\title{
High titer methyl ketone production with tailored Pseudomonas taiwanensis VLB120
}

Salome C. Nies ${ }^{1}$, Tobias B. Alter ${ }^{1}$, Sophia Nölting ${ }^{1}$, Susanne Thiery ${ }^{1}$, An N. T. Phan ${ }^{1}$, Noud Drummen ${ }^{1}$, Jay D. Keasling ${ }^{2-9}$, Lars M. Blank ${ }^{1}$, Birgitta E. Ebert ${ }^{1,10,11^{* \dagger}}$

${ }^{1}$ iAMB-Institute of Applied Microbiology, ABBt-Aachen Biology and Biotechnology, RWTH Aachen University, DE

2 Joint BioEnergy Institute, Emeryville, CA 94608, USA.

${ }^{3}$ Novo Nordisk Foundation Center for Biosustainability, Technical University of Denmark, 2800 Lyngby, DK

${ }^{4}$ Lawrence Berkeley National Laboratory, Biological Systems and Engineering Division, Berkeley, CA 94720, USA.

${ }^{5}$ Virtual Institute of Microbial Stress and Survival, Lawrence Berkeley National Laboratory, Berkeley, CA 94720, USA

${ }^{6}$ Physical Biosciences Division, Lawrence Berkeley National Laboratory, Berkeley, CA 94720, USA

7 Dept. of Bioengineering, University of California, Berkeley, CA 94720, USA

${ }^{8}$ Dept. of Chemical Engineering, University of California, Berkeley, CA 94720, USA

${ }^{9}$ Synthetic Biochemistry Center, Institute for Synthetic Biology, Shenzhen Institutes for Advanced Technologies, Shenzhen, China

${ }^{10}$ Australian Institute for Bioengineering and Nanotechnology (AIBN), the University of Queensland, Brisbane, QLD 4072, Australia

${ }^{11}$ CSIRO Future Science Platform in Synthetic Biology, Commonwealth Scientific and Industrial Research Organisation (CSIRO), Black Mountain, ACT 2601, Australia

† Corresponding author: birgitta.ebert@uq.edu.au 


\begin{abstract}
Methyl ketones present a group of highly reduced platform chemicals industrially produced from petroleum-derived hydrocarbons. They find applications in the fragrance, flavor, pharmacological, and agrochemical industries, and are further discussed as biodiesel blends. In recent years, intense research has been carried out to achieve sustainable production of these molecules by re-arranging the fatty acid metabolism of various microbes. One challenge in the development of a highly productive microbe is the high demand for reducing power. Here, we engineered Pseudomonas taiwanensis VLB120 for methyl ketone production as this microbe has been shown to sustain exceptionally high $\mathrm{NAD}(\mathrm{P}) \mathrm{H}$ regeneration rates. The implementation of published strategies resulted in $2.1 \mathrm{~g} \mathrm{Laq}^{-1}$ methyl ketones in fed-batch fermentation. We further increased the production by eliminating competing reactions suggested by metabolic analyses. These efforts resulted in the production of $9.8 \mathrm{~g} \mathrm{Laq}^{-1}$ methyl ketones (corresponding to $69.3 \mathrm{~g} \mathrm{Lorg}^{-1}$ in the in situ extraction phase) at $53 \%$ of the maximum theoretical yield. This represents a 4-fold improvement in product titer compared to the initial production strain and the highest titer of recombinantly produced methyl ketones reported to date. Accordingly, this study underlines the high potential of $P$. taiwanensis VLB120 to produce methyl ketones and emphasizes model-driven metabolic engineering to rationalize and accelerate strain optimization efforts.
\end{abstract}

Keywords: biodiesel, Pseudomonads, thioesterase, metabolic modeling, metabolic engineering, synthetic biology

Abbreviations: aq, aqueous phase; CDW, cell dry weight; DO, dissolved oxygen; GC, gas chromatography; GLC, glucose; HPLC, high-performance liquid chromatography; MiMBI, Minimization of Metabolites Balances; $\mathrm{MK}$, methyl ketone; $\mathrm{MS}$, mass spectrometry; $\mathrm{OD}_{600 \mathrm{~nm}}$, optical density determined at $600 \mathrm{~nm}$; org, organic phase; (p)FBA, (parsimonious) flux balance analysis

\title{
1. Introduction
}

The conversion of renewable feedstocks or waste streams by microbial fatty acid biosynthesis into commodity oleochemicals and transportation fuels represents a sustainable alternative to petroleum or plant-oil based processes. The range of molecules derived from fatty acids includes free fatty acids, esters, alcohols, aldehydes, ketones, alkanes, olefins, or waxes (Kim et al., 2019; Xu et al., 2016). These molecules find applications as (advanced) biofuels, flavors, fragrances, detergents, lubricants, surfactants, or plastic monomers (Buist, 2010; Lennen and Pfleger, 2013; Tee et al., 2014) and are derived from either fatty acyl Coenzyme A (CoA) or fatty acyl-acyl carrier protein thioesters. The essentiality of these metabolites for lipid biosynthesis in most living organisms (Janssen and Steinbüchel, 2014; López-Lara and Geiger, 2010) guarantees constitutive and substantial production, and successful examples show the possibility of rewiring microbial metabolism for the synthesis of fatty acid-derived products at high titers (Kim et al., 2019; Xu et al., 2016). 
Fatty acid-derived methyl ketones are garnering attention for use as flavors, fragrances, insecticides or insect repellents, solvents as well as for their promising fuel properties (Goh et al., 2012; Kimps et al., 2011; Longo, 2006). The cetane number describes the ignition behavior of diesel fuels (Dahmen and Marquardt, 2015) and has been determined for a mixture of medium-chain length methyl ketones (C11 and C13) to be around 58 (Goh et al., 2012). While this is already in the diesel range, the cetane number can be further increased to 80 to 90 by chemical modification of methyl ketones to dioxolanes (Harrison and Harvey, 2018). Microbes do not naturally synthesize methyl ketones, but recombinant production of medium-chain length congeners has been achieved in several species (Dong et al., 2019; Goh et al., 2012; Hanko et al., 2018; Müller et al., 2013; Park et al., 2012). However, most reported titers are in the $\mathrm{mg} \mathrm{L}^{-1}$ range, and lower gram-scale production has only been reported for an intensively engineered $E$. coli in a 200-h fed-batch cultivation (Goh et al., 2018).

Besides overexpression of the product biosynthesis pathway, a typical strategy to boost the synthesis of fatty acid derivatives is engineering the supply of the precursors acetyl-CoA and malonyl-CoA. This can be achieved, e.g., by overexpressing acetyl-CoA carboxylase, catalyzing the first committed step of fatty acid synthesis (Davis et al., 2000) or deletion of acetyl-CoA consuming pathways. Indeed, abolishing acetyl-CoA conversion to acetate was crucial for improving methyl ketone production in E. coli. (Goh et al., 2014). Secretion of fermentative byproducts is not only a loss of carbon but also of electrons. The latter is vital since fatty acid synthesis has a high demand for NADPH, and the supply of electrons via reduced redox coenzymes can limit the production rate. The formation of reducing equivalents can be shifted to NADPH by overexpression of a transhydrogenase (Sanchez et al., 2006), NADH kinase (Lee et al., 2009) or by changing the cofactor dependency of key enzymes. While such interventions positively impacted methyl ketone production (Goh et al., 2018), they do not increase the overall reductive power of the cell. Redox cofactor availability, therefore, remains a nontrivial challenge.

In this study, we decided on utilizing $P$. taiwanensis VLB120, an obligate aerobic bacterium as host for methyl ketone production. Pseudomonads are gaining increasing interest in industrial biotechnology due to their fast growth, solvent tolerance, and versatile metabolism (Kohler et al., 2013; Poblete-Castro et al., 2012a). Relevant for the envisaged application, Pseudomonads produce no fermentative byproducts and can sustain an exceptionally high redox cofactor regeneration rate depending on the metabolic demand (Blank et al., 2008; Ebert et al., 2011; Rühl et al., 2009). The natural ability of $P$. taiwanensis VLB120 to utilize glycerol, xylose (Kohler et al., 2015), and aromatic compounds found in lignocellulosic biomass hydrolysate (Panke et al., 1998; Poblete-Castro et al., 2012a) renders the strain interesting for biorefinery approaches.

We argue hence that $P$. taiwanensis VLB120 offers excellent traits for the production of methyl ketones. To prove its potential, we first implemented the methyl ketone synthesis pathway previously established in E. coli (Goh et al., 2012; Goh et al., 2014) and applied a metabolic model-supported engineering approach that boosted methyl ketone titer to $9.8 \mathrm{~g}$ $\mathrm{Laq}^{-1}$ and a yield of $53 \%$ of the theoretical maximum. 


\section{Material and Methods}

\subsection{Strains, media, and culture conditions}

Table 1 lists the bacterial strains used in this study. Strains were propagated in Lysogeny Broth (LB) containing $10 \mathrm{~g} \mathrm{~L}^{-1}$ peptone, $5 \mathrm{~g} \mathrm{~L}^{-1}$ sodium chloride, and $5 \mathrm{~g} \mathrm{~L}^{-1}$ yeast extract (Sambrook, 1982). Cetrimide agar (Sigma-Aldrich, St. Louis, MO, USA) was used after the mating procedures to select for Pseudomonas. Solid LB was prepared by adding $1.5 \%(\mathrm{w} / \mathrm{v})$ agar to the liquid medium. For plasmid maintenance, the gene deletion procedure, and genomic integrations, antibiotics were added to the medium as required. Gentamycin, kanamycin, and ampicillin were used at concentrations of $25 \mathrm{mg} \mathrm{L}^{-1}, 50 \mathrm{mg} \mathrm{L}^{-1}$, and $100 \mathrm{mg} \mathrm{L}^{-1}$, respectively. E. coli was grown at $37^{\circ} \mathrm{C}$, Pseudomonas at $30^{\circ} \mathrm{C}$ on a horizontal rotary shaker with a throw of $50 \mathrm{~mm}$ and a frequency of $300 \mathrm{rpm}$ (Infors, Bottmingen, Switzerland). The chemicals used in this work were obtained from Carl Roth (Karlsruhe, Germany), Sigma-Aldrich (St. Louis, MO, USA), or Merck (Darmstadt, Germany) unless stated otherwise.

Table 1. Bacterial strains and plasmids used in this study.

\begin{tabular}{|c|c|c|}
\hline Strain/Plasmid & Relevant characteristics $^{a}$ & Reference \\
\hline \multicolumn{3}{|l|}{ Plasmids } \\
\hline pEG1675 & $\begin{array}{l}\text { ColE1 derivative Bgl Brick vector containing the expression } \\
\text { cassettes araC-PBAD-fadR-fadD-Ptrc-fadM fragment and Placuv5-- } \\
\text { 'tesA-fadB-co_aco, } \mathrm{Km}^{\mathrm{R}}\end{array}$ & (Goh et al., 2014) \\
\hline pTn-1 & $\begin{array}{l}\text { E. coli/Pseudomonas shuttle vector with nagR/p } \mathrm{p}_{\mathrm{NagAa}} \\
\text { promoter, } \mathrm{Amp}^{\mathrm{R}}, \mathrm{Gm}^{\mathrm{R}}\end{array}$ & $\begin{array}{l}\text { (Wierckx et al., } \\
\text { 2005) }\end{array}$ \\
\hline pBNTmcs & $\begin{array}{l}\text { E. coli/Pseudomonas shuttle vector with nagR/pNagAa } \\
\text { promoter, } \mathrm{Km}^{\mathrm{R}}\end{array}$ & $\begin{array}{l}\text { (Verhoef et al., } \\
\text { 2010) }\end{array}$ \\
\hline pEMG & ori R6K, lacZ $\alpha$ with two flanking I-Scel sites, $\mathrm{Km}^{\mathrm{R}}$ & $\begin{array}{l}\text { (Martínez-García } \\
\text { and de Lorenzo, } \\
\text { 2011) }\end{array}$ \\
\hline pEMG_ko_fadA & PVLB_07895 deletion plasmid, $\mathrm{Km}^{\mathrm{R}}$ & This study \\
\hline pEMG_ko_fadE & PVLB_07065 deletion plasmid, $\mathrm{Km}^{\mathrm{R}}$ & This study \\
\hline pEMG_ko_fadE2 & PVLB_10075 deletion plasmid, $\mathrm{Km}^{\mathrm{R}}$ & This study \\
\hline pEMG_ko_fadA2 & PVLB_12575 deletion plasmid, $\mathrm{Km}^{\mathrm{R}}$ & This study \\
\hline pEMG_ko_pha & PVLB_02155-02165 deletion plasmid, $\mathrm{Km}^{\mathrm{R}}$ & This study \\
\hline pEMG_ko_tesB & PVLB_03305 deletion plasmid, $\mathrm{Km}^{\mathrm{R}}$ & This study \\
\hline pTn7-M & ori $\mathrm{R} \overline{6} \mathrm{~K}$, terminal sequences of $\mathrm{Tn} 7(\mathrm{Tn} 7 \mathrm{~L}$ and $\mathrm{Tn} 7 \mathrm{R}), \mathrm{Km}^{\mathrm{R}} \mathrm{Gm}^{\mathrm{R}}$ & (Zobel et al., 2015) \\
\hline pTn7-MK & 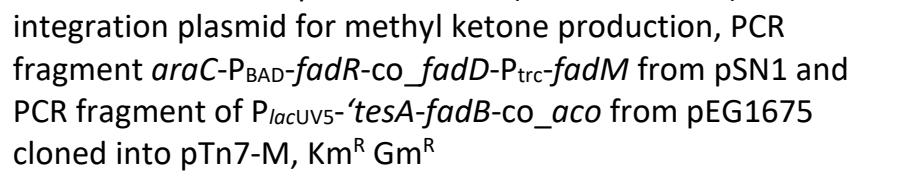 & This study \\
\hline pSN1 & 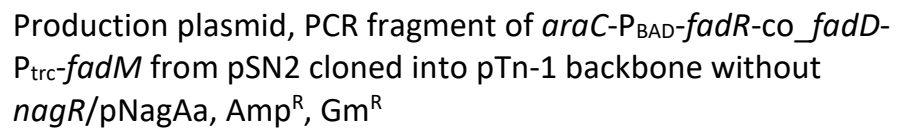 & This study \\
\hline pSN2 & $\begin{array}{l}\text { Production plasmid, PCR fragment PlacUv5-'tesA-fadB-co_aco } \\
\text { from pEG1675 cloned into pBNTmcs backbone without } \\
\text { nagR/pNagAa promoter, } \mathrm{Km}^{\mathrm{R}}\end{array}$ & This study \\
\hline \multicolumn{3}{|l|}{ E. coli } \\
\hline $\mathrm{DH} 5 \alpha$ & $\begin{array}{l}\text { supE44, } \Delta / a c U 169(\varphi 80 / a c Z \Delta M 15), \text { hsdR17 }\left(\mathrm{rk}^{-} \mathrm{mk}^{+}\right) \text {, recA1, } \\
\text { endA1, gyrA96, thi-1, relA1 }\end{array}$ & (Hanahan, 1985) \\
\hline DH10beta & $\begin{array}{l}\text { DH10B derivative, T1 phage resistant, endonuclease I (endA1) } \\
\text { deficient; used for plasmid preparations }\end{array}$ & New England Biolabs \\
\hline $\mathrm{DH} 5 \alpha \lambda$ pir1 & $\begin{array}{l}\text { Apir lysogen of } \mathrm{DH} 5 \alpha \text {; host for oriV(R6K) vectors at high copy } \\
\text { numbers }\end{array}$ & $\begin{array}{l}\text { Thermo Fisher } \\
\text { Scientific }\end{array}$ \\
\hline
\end{tabular}




\begin{tabular}{|c|c|c|}
\hline One Shot PIR2 & $\begin{array}{l}\mathrm{F}^{-} \Delta / a c 169 \text { rpoS(Am) robA1 creC510 hsd } \mathrm{R} 514 \text { endA recA1 } \\
\text { uidA(AMlul)::pir }\end{array}$ & $\begin{array}{l}\text { Thermo Fisher } \\
\text { Scientific }\end{array}$ \\
\hline HB101 pRK2013 & $\begin{array}{l}\text { hsdR- } \mathrm{M}^{+} \text {, proA2, leuB6, thi-1, recA; conjugation helper strain, } \\
\text { bears plasmid pRK2013, } \mathrm{Gm}^{\mathrm{R}}\end{array}$ & (Ditta et al., 1980) \\
\hline $\mathrm{DH} 5 \alpha \mathrm{pSW}-2$ & $\begin{array}{l}\mathrm{DH} 5 \alpha \text { bearing } \mathrm{pSW}-2 \text { (ori RK2, } x y / S, P m \rightarrow \mathrm{I}-\mathrm{Scel} \\
\text { (transcriptional fusion of I-Scel to } \mathrm{Pm} \text { ), } \mathrm{Gm}^{\mathrm{R}}\end{array}$ & $\begin{array}{l}\text { (Martínez-García } \\
\text { and de Lorenzo, } \\
\text { 2011) }\end{array}$ \\
\hline $\begin{array}{l}\text { DH5 } \alpha \text { גpir pTNS1 } \\
\text { P. taiwanensis }\end{array}$ & DH5 $\alpha \lambda$ pir bearing pTNS1; ori R6K, TnSABC+D operon, $A_{m p}{ }^{R}$ & de Lorenzo lab \\
\hline VLB120 & $\begin{array}{l}\text { wild type isolated from forest soil in the Stuttgart area (DE), } \\
\text { carrying the native megaplasmid pSTY. }\end{array}$ & $\begin{array}{l}\text { Prof. Andreas } \\
\text { Schmid, UFZ Leipzig, } \\
\text { DE }\end{array}$ \\
\hline VLB120 pProd & $\begin{array}{l}\text { wild type with production plasmids pSN1 and pSN2 (pSN1 + } \\
\text { pSN2 = pProd) }\end{array}$ & This study \\
\hline VLB120 $\Delta 3$ pProd & $\begin{array}{l}\text { VLB120 with deletion of fadA (PVLB_07895), fadE } \\
\text { (PVLB_07065), and fadE2 (PVLB_10075), carrying pSN1 and } \\
\text { pSN2, pSTY }\end{array}$ & This study \\
\hline VLB120 $\Delta 3$ attTn7::MK & VLB120 $\Delta 3$ with genomic integration of pTn7-MK, pSTY & This study \\
\hline $\begin{array}{l}\text { VLB120 } \Delta 3 \Delta \text { pha } \\
\text { pProd }\end{array}$ & $\begin{array}{l}\text { VLB120 } \Delta 3 \text { with deletion of the pha operon (PVLB_02155- } \\
\text { 02165), carrying pSN1 and pSN2, pSTY }\end{array}$ & This study \\
\hline $\begin{array}{l}\text { VLB120 } \triangle 3 \triangle \text { fadA2 } \\
\text { pProd }\end{array}$ & $\begin{array}{l}\text { VLB120 } \triangle 3 \text { with deletion of fadA2 (PVLB_12575), carrying } \\
\text { pSN1 and pSN2, pSTY' }\end{array}$ & This study \\
\hline $\begin{array}{l}\text { VLB120 } \Delta 3 \Delta t e s B \\
\text { pProd }\end{array}$ & $\begin{array}{l}\text { VLB120 } \Delta 3 \text { with } \Delta \text { tesB (PVLB_03305), with pSN1 and pSN2, } \\
\text { pSTY }\end{array}$ & This study \\
\hline VLB120 $\Delta 6$ pProd & $\begin{array}{l}\text { VLB120 } \triangle 3 \text { with deletion of fadA2 (PVLB_12575), the pha } \\
\text { operon (PVLB_02155-02165), and tesB (PVLB_03305), carrying } \\
\text { pSN1 and pSN2, pSTY- }\end{array}$ & This study \\
\hline
\end{tabular}

${ }^{a} \mathrm{Amp}^{\mathrm{R}}, \mathrm{Km}^{\mathrm{R}}, \mathrm{Gm}^{\mathrm{R}}$ : ampicillin, kanamycin, and gentamycin resistance, respectively.

Methyl ketone production by the engineered Pseudomonas was characterized by cultivating the strains in 500-mL flasks with $10 \%$ (v/v) mineral salt medium (Hartmans et al., 1989) containing $7.76 \mathrm{~g} \mathrm{~L}^{-1} \mathrm{~K}_{2} \mathrm{HPO}_{4}, 3.26 \mathrm{~g} \mathrm{~L}^{-1} \mathrm{NaH}_{2} \mathrm{PO}_{4}, 2 \mathrm{~g} \mathrm{~L}^{-1}\left(\mathrm{NH}_{4}\right)_{2} \mathrm{SO}_{4}, 0.1 \mathrm{~g} \mathrm{~L}^{-1} \mathrm{MgCl}_{2} \cdot 6 \mathrm{H}_{2} \mathrm{O}, 10$ $\mathrm{mg} \mathrm{L}^{-1}$ EDTA, $2 \mathrm{mg} \mathrm{L}^{-1} \mathrm{ZnSO}_{4} \cdot 7 \mathrm{H}_{2} \mathrm{O}, 1 \mathrm{mg} \mathrm{L}^{-1} \mathrm{CaCl}_{2} \cdot 2 \mathrm{H}_{2} \mathrm{O}, 5 \mathrm{mg} \mathrm{L}^{-1} \mathrm{FeSO}_{4} \cdot 7 \mathrm{H}_{2} \mathrm{O}, 0.2 \mathrm{mg} \mathrm{L}^{-1}$ $\mathrm{Na}_{2} \mathrm{MoO}_{4} \cdot 2 \mathrm{H}_{2} \mathrm{O}, 0.2 \mathrm{mg} \mathrm{L}^{-1} \mathrm{CuSO}_{4} \cdot 5 \mathrm{H}_{2} \mathrm{O}, 0.4 \mathrm{mg} \mathrm{L}^{-1} \mathrm{CoCl}_{2} \cdot 6 \mathrm{H}_{2} \mathrm{O}, 1 \mathrm{mg} \mathrm{L}-1 \mathrm{MnCl}_{2} \cdot 2 \mathrm{H}_{2} \mathrm{O}$ supplemented with $25 \mathrm{mM}$ or $50 \mathrm{mM}$ glucose unless stated otherwise. For continuous in situ extraction of methyl ketones, $12.5 \mathrm{~mL} n$-decane was added as an overlay. If not indicated otherwise samples for analytical measurements were taken after $24 \mathrm{~h}$ and $48 \mathrm{~h}$. Before inoculating the preculture, LB agar plates (with appropriate antibiotics) were streaked from frozen glycerol stocks and were incubated overnight at $30^{\circ} \mathrm{C}$. A single colony was used to inoculate $5 \mathrm{~mL}$ of mineral salt medium containing antibiotics, supplemented with $25 \mathrm{mM}$ glucose in a $15-\mathrm{mL}$ glass tube. The preculture was transferred twice before inoculating the main culture. The main cultures were inoculated from liquid precultures to an approximate optical density at $600 \mathrm{~nm}\left(\mathrm{OD}_{600 \mathrm{~nm}}\right)$ of 0.1 . Gene expression was induced after 5-7 $\mathrm{h}$ with the addition of $2 \mathrm{mM}$ IPTG and $1 \mathrm{mM}$ arabinose for plasmid-bearing strains. Arabinose and IPTG were added to a final concentration of $1 \mathrm{mM}$ for gene expression in strains with a genome integrated methyl ketone pathway. All experiments were performed in biological duplicates unless stated otherwise. 


\subsection{Plasmid construction and transformation}

Primers were ordered as unmodified DNA oligonucleotides from Eurofins Genomics (Ebersberg, Germany) and are listed in Supplementary Table S1. The Monarch Plasmid Miniprep Kit (New England Biolabs, Ipswich, MA, USA) was used to isolate plasmids, which were either digested with restriction enzymes purchased from New England Biolabs (Ipswich, MA, USA) or were used as a template for PCR using Q5 High-Fidelity Polymerase (New England Biolabs, Ipswich, MA, USA). The plasmid pEG1675 developed by Goh et al. (Goh et al., 2014) for methyl ketone production in $E$. coli served as a template for the amplification of two genetic cassettes required for methyl ketone overproduction. All genes, except co_aco, originate from E. coli; co fadD had been codon-optimized for high expression in E. coli. 'tesA encodes a truncated variant of the thioesterase TesA that expresses in the cytosol; co_aco designates the acyl-CoA oxidase encoding gene Mlut_11700 from Micrococcus luteus codonoptimized for E. coli. The plasmids pSN1 and pSN2 were introduced into chemically competent E. coli DH10beta (New England Biolabs, Ipswich, MA, USA). Transformants were screened by colony PCR using OneTaq 2x Master Mix (New England Biolabs, Ipswich, MA, USA). Production plasmids were isolated and analyzed by PCR and Sanger sequencing (Eurofins Genomics, Germany).

For gene deletions, upstream (TS1) and downstream (TS2) regions with a length of 400-800 bp flanking the specific target gene were amplified using Q5 High-Fidelity Polymerase (New England Biolabs, Ipswich, MA, USA). Genomic DNA of $P$. taiwanensis VLB120 was used as the template for the PCR and was isolated using the High Pure PCR Template Preparation Kit (Hoffmann-La-Roche, Basel, Switzerland). Plasmids were constructed by Gibson assembly using the NEBuilder Hifi DNA Assembly Master Mix (New England Biolabs, Ipswich, MA, USA). pEMG plasmids were transformed into competent $E$. coli $\mathrm{DH} 5 \alpha \lambda$ pir1 via electroporation (Choi et al., 2006), and the correct assembly was confirmed by colony PCR. The cell material was lysed in alkaline polyethylene glycol for enhanced colony PCR efficiency as described elsewhere (Chomczynski and Rymaszewski, 2006).

\subsection{Generation of deletion strains and genomic integration}

Targeted gene deletions were performed using the I-Scel-based system developed by Martinez-Garcia and de Lorenzo (Martínez-García and de Lorenzo, 2011) and as described previously (Wynands et al., 2018). Briefly, the conjugational transfer of the mobilizable knockout plasmid from E. coli $\mathrm{DH} 5 \alpha$ p pir1 to Pseudomonas was performed via patch mating overnight (Wynands et al., 2018). After conjugation, the pSW-2 plasmid encoding the I-Scelendonuclease was conjugated into Pseudomonas co-integrates. The induction of I-Scel expression with 3-methyl benzoate was omitted as the basal expression level was sufficient. Kanamycin-sensitive clones were directly isolated, positive clones were cured of pSW-2, and re-streaked several times. The gene deletion was confirmed by colony PCR as described above and by Sanger sequencing.

Genomic integration into the attTn7 of Pseudomonas was carried out as described previously (Zobel et al., 2015). Briefly, patch mating was carried out with E. coli pRK2013 (helper strain), E. coli DH5 $\alpha-\lambda$ pir pTnS-1 (strain leading transposase), E. coli DH5 $\alpha$ One Shot PIR 2 pTn7-MK 
and VLB120 $\Delta 3$. After $48 \mathrm{~h}$ of conjugation at $30^{\circ} \mathrm{C}$, cell material was selected on cetrimide agar containing gentamycin. Colonies were screened for positive insertion with colony PCR as described above.

\subsection{Analysis of cell growth and sugar metabolism during aqueous organic two-phase cultivations}

Samples taken from aqueous organic two-phase cultivations with $n$-decane were treated as follows. First, the different phases were separated by centrifugation for $5 \mathrm{~min}$ at 17,000 $\mathrm{xg}$, and the interphase was marked. The organic phase was removed for gas chromatography (GC) analysis, whereas the remaining aqueous phase was filtered through a $0.2 \mu \mathrm{m}$ membrane filter and stored at $-20^{\circ} \mathrm{C}$ until further high-performance liquid chromatography (HPLC) analysis. As $n$-decane would perturb $\mathrm{OD}_{600 \mathrm{~nm}}$ measurements, residual $n$-decane was carefully removed from the inner tube wall with a cellulose tissue. The cell pellet was resuspended in $0.9 \%(\mathrm{w} / \mathrm{v}) \mathrm{NaCl}$ to a final volume equaling the initial aqueous phase. The $\mathrm{OD}_{600 \mathrm{~nm}}$ was determined in technical triplicates using an Ultrospec 10 spectrophotometer (GE Healthcare, Chicago, IL, USA).

Glucose and gluconate concentrations were determined by HPLC. The analysis was performed using a Beckman System Gold 126 Solvent Module equipped with a System Gold 166 UVdetector (Beckman Coulter) and a Smartline RI detector 2300 (KNAUER Wissenschaftliche Geräte, Berlin, Germany). Analytes were separated on the organic resin column Metab AAC (ISERA, Düren, Germany) eluted with $5 \mathrm{mM} \mathrm{H}_{2} \mathrm{SO}_{4}$ at an isocratic flow of $0.6 \mathrm{~mL} \mathrm{~min}^{-1}$ at $40{ }^{\circ} \mathrm{C}$ for $40 \mathrm{~min}$. Glucose and gluconate were analyzed using the RI detector, whereas gluconate was determined with the UV detector at a wavelength of $210 \mathrm{~nm}$.

\subsection{Methyl ketone analysis with gas chromatography}

Quantification of methyl ketones was performed on a Trace GC Ultra (Thermo Scientific, Waltham, MA, USA) equipped with a flame ionization detector (FID). Analyte separation was achieved with a polar ZB-WAX column (30 m length, $0.25 \mathrm{~mm}$ inner diameter, $0.25 \mu \mathrm{m}$ film thickness, Zebron, Phenomenex, UK) and a constant helium flow of $2 \mathrm{~mL} \mathrm{~min}^{-1}$. The initial oven temperature of $80^{\circ} \mathrm{C}$ was held for $2.5 \mathrm{~min}$, increased to $250^{\circ} \mathrm{C}$ at $20^{\circ} \mathrm{C} \mathrm{min}^{-1}$ and held constant for $10 \mathrm{~min}$. The injection volume was $1 \mu \mathrm{L}$, and the injector temperature was set to $250^{\circ} \mathrm{C}$. The split ratio was adjusted depending on the analyte concentration. External standard quantification with 2-undecanone (Alfa Aesar, Ward Hill, MA, USA), 2-tridecanone (Alfa Aesar, Ward Hill, MA, USA), 2-pentadecanone, and 2-heptadecanone was performed. No authentic standards were available for the monounsaturated methyl ketones (C11:1, C13:1, C15:1, and C17:1). Those congeners were first identified by GC-mass spectrometry (MS) analysis using a TRACE GC Ultra coupled to the triple quadrupole TSQ 8000 (Thermo Fisher Scientific) and identified by comparing the mass spectra with published data (Goh et al., 2012) (Supplementary Figure S1). The tuning and calibration of the mass spectrometer were done prior to analysis. A $1 \mu \mathrm{L}$ aliquot of the sample was injected into a VF-5ms capillary column (30 m × $250 \mu \mathrm{m}$ i.d., $0.25 \mu \mathrm{m}$ film thickness) with a $10 \mathrm{~m}$ EZ-Guard column (Agilent). The injection temperature was $250{ }^{\circ} \mathrm{C}$, and the helium carrier gas flow was set to $1 \mathrm{~mL} / \mathrm{min}$. The column temperature was held at $80^{\circ} \mathrm{C}$ for $2 \mathrm{~min}$, increased by $80^{\circ} \mathrm{C} \mathrm{min}^{-1}$ to $120^{\circ} \mathrm{C}$, 
increased by $15^{\circ} \mathrm{C} \mathrm{min}-1$ to $250{ }^{\circ} \mathrm{C}$ and then increased by $100{ }^{\circ} \mathrm{C} \mathrm{min}^{-1}$ to $325^{\circ} \mathrm{C}$, at which it was held constant for $3 \mathrm{~min}$. The transfer line and ion source temperatures were $280^{\circ} \mathrm{C}$ and $300{ }^{\circ} \mathrm{C}$, respectively. Ions were generated by electron ionization at $70 \mathrm{eV}$.

Saturated methyl ketones were quantified with a calibration curve recorded using authentic standards. We assumed the same response factor for the monounsaturated methyl ketones as for their unsaturated counterparts and quantified the concentration using the respective calibration curve. Methyl ketone titers were related to the aqueous phase, as reported previously (Hanko et al., 2018). For batch fermentations, the aqueous concentration $c_{a q}$ at time point $t_{i}\left(c_{a q, t_{i}}\right)$ was calculated using equation 1 :

$$
c_{a q, t_{i}}=c_{\text {org }, t_{i}} \cdot \frac{V_{\text {org }, t_{i}}}{V_{a q, t_{i}}}=c_{\text {org }, t_{i}} \cdot \frac{V_{\text {org }, t_{i-1}}-V_{\text {sample }, t_{i}} \cdot \frac{V_{\text {org }, t_{i-1}}}{V_{a q, t_{i-1}}}}{V_{a q, t_{i-1}}-V_{\text {sample }, t_{i}} \cdot\left(1-\frac{V_{\text {org }, t_{i-1}}}{V_{a q, t_{i-1}}}\right)}
$$

with $c_{\text {org }, t_{i}}$ being the experimentally determined methyl ketone concentration, and $V_{\text {org }, t_{i}}$, $V_{o r g, t_{i}-1}$ and $V_{a q, t_{i}}, V_{a q, t_{i}}$ the volume of the organic, respectively the aqueous phase at time point $t_{i}$ or $t_{i-1}$. For fed-batch cultivations, the change in the fermentation volume due to the aqueous medium feed $F_{\text {feed }}$ needed to be considered. For this fermentation mode, the aqueous concentration was calculated using equation 2 .

$$
c_{a q, i}=c_{o r g, i} \cdot \frac{V_{o r g, t_{i-1}}-V_{\text {sample }, t_{i}} \cdot \frac{V_{\text {org } t_{i-1}}}{V_{a q, t_{i-1}}+F_{\text {feed }} \cdot\left(t-t_{i-1}\right)}}{V_{a q, t_{i-1}}+F_{f e e d} \cdot\left(t_{i}-t_{i-1}\right)-V_{\text {sample } t_{i}} \cdot\left(1-\frac{V_{\text {org }, t_{i-1}}}{V_{a q, t_{i-1}}}\right)}
$$

\subsection{Bioreactor cultivations for methyl ketone production}

Bioreactor cultivations were performed in 1.3-L fermenters (BioFlow120, Eppendorf, Germany) with a working volume of $0.5 \mathrm{~L}$ mineral salt medium $(0.4 \mathrm{~L}$ mineral salt medium in fed-batch), supplemented with $2 \%(\mathrm{w} / \mathrm{v})$ glucose monohydrate and $100 \mathrm{~mL} n$-decane $(17 \%$ $(\mathrm{v} / \mathrm{v}))$. The temperature was maintained at $30^{\circ} \mathrm{C}$; the $\mathrm{pH}$ was controlled at a value of 7 with 2 $\mathrm{M} \mathrm{KOH}$ and $25 \% \mathrm{NH}_{4} \mathrm{OH}$ in batch and fed-batch cultivations, respectively, and $4 \mathrm{M} \mathrm{H}_{2} \mathrm{SO}_{4}$. The dissolved oxygen (DO) level was kept above $30 \%$ using a stirring cascade. After 6-7 h inoculation, the gene expression was induced with $2 \mathrm{mM}$ IPTG and $1 \mathrm{mM}$ arabinose. For the fed-batch cultivation, the DO signal was used to start the feeding pump, when DO signal exceeded $70 \%$, the feeding pump started with $5 \mathrm{~mL} \mathrm{~h}^{-1}$, with a feeding medium of $585 \mathrm{mM}$ glucose, $7.76 \mathrm{~g} \mathrm{~L}^{-1} \mathrm{~K}_{2} \mathrm{HPO}_{4}, 3.26 \mathrm{~g} \mathrm{~L}^{-1} \mathrm{NaH}_{2} \mathrm{PO}_{4}, 2 \mathrm{~g} \mathrm{~L}^{-1}\left(\mathrm{NH}_{4}\right)_{2} \mathrm{SO}_{4}, 0.3 \mathrm{~g} \mathrm{~L}^{-1} \mathrm{MgCl}_{2} \cdot 6 \mathrm{H}_{2} \mathrm{O}, 30 \mathrm{mg}$ $\mathrm{L}^{-1}$ EDTA, $6 \mathrm{mg} \mathrm{L}^{-1} \mathrm{ZnSO}_{4} \cdot 7 \mathrm{H}_{2} \mathrm{O}, 3 \mathrm{mg} \mathrm{L}^{-1} \mathrm{CaCl}_{2} \cdot 2 \mathrm{H}_{2} \mathrm{O}, 15 \mathrm{mg} \mathrm{L}^{-1} \mathrm{FeSO}_{4} \cdot 7 \mathrm{H}_{2} \mathrm{O}, 0.6 \mathrm{mg} \mathrm{L}^{-1}$ $\mathrm{Na}_{2} \mathrm{MoO}_{4} \cdot 2 \mathrm{H}_{2} \mathrm{O}, 0.6 \mathrm{mg} \mathrm{L}^{-1} \mathrm{CuSO}_{4} \cdot 5 \mathrm{H}_{2} \mathrm{O}, 1.2 \mathrm{mg} \mathrm{L}^{-1} \mathrm{CoCl}_{2} \cdot 6 \mathrm{H}_{2} \mathrm{O}, 3 \mathrm{mg} \mathrm{L}^{-1} \mathrm{MnCl}_{2} \cdot 2 \mathrm{H}_{2} \mathrm{O}, 2 \mathrm{mM}$ IPTG, $1 \mathrm{mM}$ arabinose, and appropriate antibiotics (gentamycin and kanamycin). The culture broth was sampled regularly for $\mathrm{OD}_{600 \mathrm{~nm}}$ determination, extracellular metabolite analysis, and methyl ketone quantification. 


\subsection{Model-guided strain-design}

For the model-guided strain design, the genome-scale metabolic model iJN1411 of Pseudomonas putida KT2440 (Nogales et al., 2017) was employed and manually adapted to match the enzymatic repertoire of $P$. taiwanensis VLB120 (Supplementary file S1). In silico synthesis of methyl ketone precursors was enabled by extending the model with a thioesterase III reaction ( $f a d M)$ converting $\beta$-ketoacyl-CoA to the respective $\beta$-keto acid for each methyl ketone congeners (C11-C17). Additional spontaneous reactions modeled the cleavage of $\mathrm{CO}_{2}$ from the $\beta$-keto acid yielding the specific methyl ketones, which are transported to the extracellular space by additional passive transport reactions. The exchange of all considered methyl ketones was pooled in a single reaction with fixed stoichiometric coefficients for each congener. The coefficients were calculated based on the final molar concentration ratios of the most dominant methyl ketones produced by the VLB120 $\Delta 3$ pProd (cf. Results, section 3.3). The thioesterase encoded by fadA was additionally deleted to fully resemble VLB120 $\Delta 3$ pProd. In the following, we refer to iJN1411_MK for this final metabolic reconstruction. For the prediction of genetic intervention strategies that enhance methyl ketones production, an optimization algorithm based on a genetic algorithm was applied (Alter et al., 2018). The Supplementary File S2 lists the applied optimization parameters. The genetic algorithm utilizes the principles of natural selection to evolve a random, initial population of solutions towards optimality. Each solution comprises a set of gene deletions that relate to specific reaction deletions in a stoichiometric metabolic model by its inherited gene-protein-reaction relations. The impact of a set of gene deletions on production was evaluated by using the yield $\mathrm{Y}_{\mathrm{GM}}$ of methyl ketones on glucose as the fitness function. The yield was calculated by $\mathrm{Y}_{\mathrm{GM}}=v_{\mathrm{MK}} / v_{\mathrm{GLC}}$, where $v_{\mathrm{MK}}$ and $v_{\mathrm{GLC}}$ are the flux of the methyl ketones exchange reaction and the glucose uptake rate, respectively. Both $v_{\mathrm{MK}}$ and $v_{\mathrm{GLC}}$ are variables in the flux distributions computed by the Minimization of Metabolites Balances (MiMBI) algorithm (Brochado et al., 2012). MiMBI calculates mutant flux distributions by minimizing the distance to a reference or wild-type flux distribution with respect to the metabolite balance vector, which comprises the sums of absolute flux rates of all reactions producing or consuming a particular metabolite. We derived a reference flux distribution from the phenotypic flux data of the cultivation of VLB120 $\Delta 3$ pProd (cf. Results, section 3.3) using a parsimonious flux balance analysis (pFBA) approach (Lewis et al., 2010). Since data from the exponential growth phase were used to compute the reference steady-state flux distribution, the reference MK yield is significantly lower as compared to the yield at the end of the batch cultivation. During the evolution of a population to near-optimality, genetic algorithms generate a multitude of solutions, thus simultaneously condense the full solution space to a subset of the most beneficial gene deletions. While the ultimate best set of genetic interventions is most promising for methyl ketone overproduction, alternative solutions with a similar fitness may also be valuable candidates for application. Moreover, information about the significance of gene deletions in generating an overproducing phenotype is desirable for deciding on the order by which to apply single genetic interventions. The first step to assist in this practical issue was to extract solutions generated by the genetic algorithm with an 
increase in methyl ketone yield of at least $50 \%$ compared to the VLB120 $\Delta 3$ pProd strain. All gene deletion targets were ranked according to the number of occurrences within these solutions.

\section{Results and discussion}

\subsection{Metabolic engineering of $P$. taiwanensis VLB120 for methyl ketone production}

The ability of Pseudomonas to produce methyl ketones was initially assessed following a strategy previously used in E. coli (Goh et al., 2012; Goh et al., 2014). This approach included the truncation of the $\beta$-oxidation cycle at the 3-ketothiolase (fadA deletion), the overexpression of a type III thioesterase (fadM) to drain 3-keto-acyl CoAs from the $\beta$-oxidation cycle, and ramping up fatty acid synthesis and the subsequent oxidation by overexpression of corresponding genes (Figure 1A).

(A)

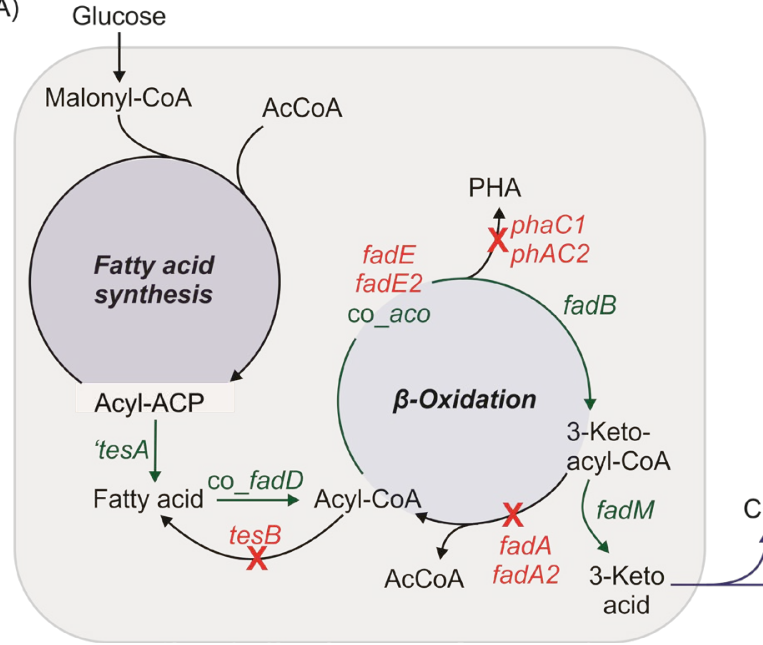

(B)

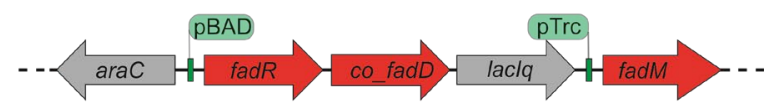

(C)

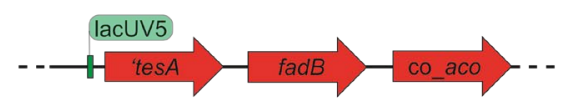

Figure 1. (A) Fatty acid biosynthesis and degradation pathways in P. taiwanensis engineered for methyl ketone production. Gene names in green indicate overexpression; those in red represent gene deletion targets. (B/C) Schematic illustration of genetic constructs used for recombinant methyl ketone production in $P$. taiwanensis. The expression cassettes (B) and (C) were either expressed from the plasmids pSN1 (B) and pSN2 (C) or as a combined construct integrated into the genome as a single copy. See text for further details.

For the overexpression of the product synthesis genes, two plasmids were constructed. Plasmid pSN1 (Figure 1B) carried the fatty acid-responsive transcription factor fadR, fadD, encoding an acyl-CoA synthetase, and fadM encoding a thioesterase III in the pTn1 backbone (Nijkamp et al., 2005; Wierckx et al., 2005). The expression of these genes was under the control of either the inducible pBAD or pTrc promoter. The truncated 'tesA gene, encoding a cytosolic thioesterase I, $\operatorname{fadB}$, a subunit of the fatty acid oxidation complex with hydratase activity, and a codon-optimized version of the acyl-CoA oxidase gene Mlut_11700 from Micrococcus luteus (co_aco) were inserted into the pBNTmcs backbone (Verhoef et al., 2010) and expressed under the control of the inducible lacUV5 promoter (plasmid pSN2, Figure 1C). The co-transformation of the wild type $P$. taiwanensis VLB120 with the two plasmids pSN1 and pSN2 resulted in VLB120 pProd, with pProd designating the two production plasmids pSN1 and pSN2. 
The two thioesterases 'TesA and FadM are characterized by a broad substrate specificity and act on molecules with carbon chain lengths between C12-C16 ('TesA) and C12-C18 (FadM), respectively (Goh et al., 2012; Nie et al., 2008; Steen et al., 2010). They were chosen to gear the methyl ketones synthesis towards congeners with 11 to 17 carbon atoms for potential use as diesel blend. This distribution was confirmed for the proof-of-concept strain VLB120 pProd, which produced in $24 \mathrm{~h} 0.6 \mathrm{mg} \mathrm{Laq}^{-1}$ total methyl ketones in a mineral salt medium and the presence of a second organic layer of $n$-decane (Figure 2). The observed distribution of methyl ketone congeners reflects the substrate specificities of the overexpressed acyl-CoA thioesterases 'TesA and FadM. The difference to the natural fatty acid profile of P. taiwanensis VLB120, which is dominated by C16:0, C16:1, and C18:1 fatty acids (Rühl et al., 2012 ) indicates that the expressed thioesterase 'TesA efficiently drains shorter chain length molecules from the fatty acid biosynthesis pathway.

We further modified the genetic background of $P$. taiwanensis VLB120 by deleting the thioesterase encoding gene fadA (Goh et al., 2012). This intervention results in a truncation of the $\beta$-oxidation cycle, which should enforce the flux towards $\beta$-keto acids and their spontaneous decarboxylation to methyl ketones in fadM expressing strains (Goh et al., 2012). Since we expressed a heterologous, soluble acyl-CoA oxidase ( $\mathrm{co}_{-} a c o$ ), we deleted the redundant, native acyl-CoA dehydrogenase encoding genes PVLB_07065 (fadE) and PVLB_10075 (here referred to as fadE2) in the engineered strain. The resulting triple knockout strain is referred to as VLB120 $\Delta 3$.

We used this basis strain to test the effect of different pathway gene expression levels by either expressing the pathway genes from the multi-copy number plasmids pSN1 and pSN2 (VLB120 $\Delta 3$ pProd) or a single-copy genome-integrated cassette (VLB120 $\Delta 3$ attTn7::MK). While the latter strain will express the pathway genes at a lower level, it might profit from a reduced metabolic burden associated with plasmid replication. To this end, the two fragments (Figure 1B\&C) were amplified from the pSN1 and pSN2 plasmids, cloned into the pTn7-M vector, and integrated into the single, genomic attTn7 site using a mini-Tn7 transposon-based method (Zobel et al., 2015).

\subsection{Characterization of methyl ketone production by plasmid-based and single-copy genome integrated pathway expression}

We aimed for a balanced expression of the pathway genes to maximize productivity and therefore determined the optimal inducer concentration for the plasmid-based and chromosomally integrated cassette. With the two-plasmid based approach, the highest titers were achieved with $1 \mathrm{mM}$ arabinose and $2 \mathrm{mM}$ IPTG, whereas $1 \mathrm{mM}$ arabinose and $1 \mathrm{mM}$ IPTG was optimal for the expression of the genome integrated single-copy methyl ketone pathway (Supplementary Figure S2).

The deletion of fadA significantly boosted methyl ketone production in these strains. In a 24-h shake flask experiment, VLB120 $\Delta 3$ pProd reached $112 \mathrm{mg} \mathrm{Laq}^{-1}$ total methyl ketones (187-fold compared to VLB120 pProd) with a yield of $24.9 \mathrm{mg} \mathrm{gGLC}^{-1}$ (Figure 2). As with its predecessor strain, monounsaturated and saturated methyl ketones with a chain length between $\mathrm{C} 11$ and C17 were detected. 
The replication of the two large production plasmids exerted a high metabolic burden on VLB120 $\Delta 3$ pProd evident from a substantial growth defect. This burden was reduced in VLB120 $\Delta 3$ attTn7::MK, which grew faster (specific growth rate $\mu=0.61 \mathrm{~h}^{-1}$ vs $0.44 \mathrm{~h}^{-1}$ ) and reached a higher maximal biomass concentration $\left(\mathrm{OD}_{600 \mathrm{~nm}}\right.$ of $\left.\sim 7 \mathrm{vs} \sim 4\right)$. Despite the growth disadvantage, plasmid-based expression of the pathway genes in VLB120 $\Delta 3$ pProd outperformed methyl ketone production in VLB120 $\Delta 3$ attTn7::MK, which accumulated a total methyl ketone titer of only $18 \mathrm{mg} \mathrm{Laq}^{-1}$. For this reason, we continued all further work with VLB120 $\Delta 3$ pProd.

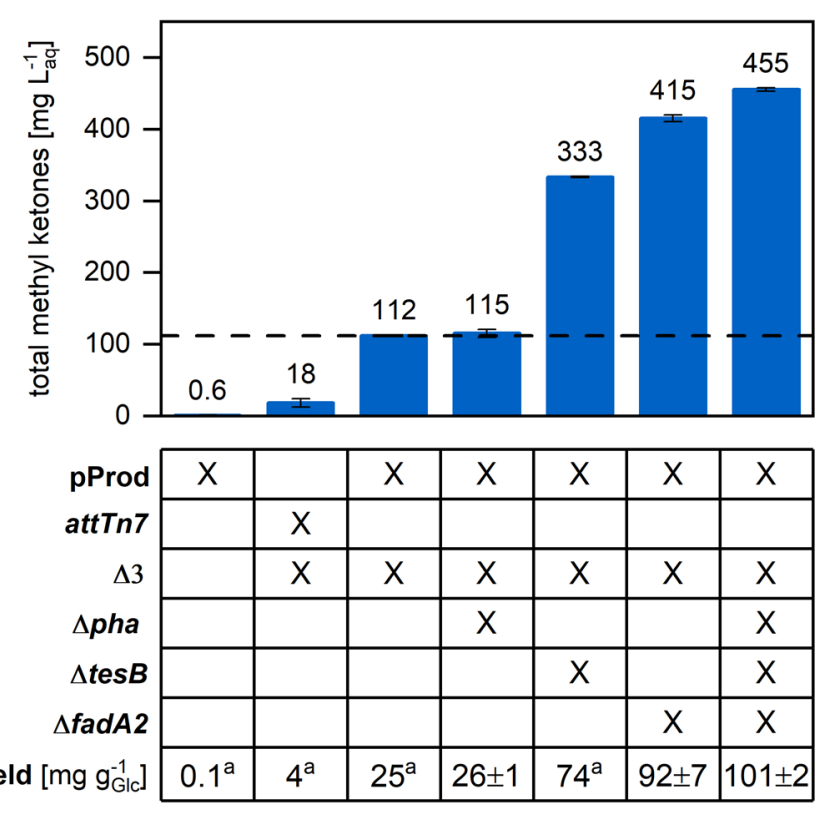

Figure 2. Methyl ketone production in metabolically engineered $P$. taiwanensis VLB120 strains. attTn7 refers to the strain with genome integrated production pathway genes; $\Delta 3$ summarizes the deletions of $\operatorname{fad} A, \operatorname{fad} E$, and fadE2; $\triangle p h a$ comprises the deletion of the pha operon (polyhydroxyalkanoate synthases and depolymerase). The dotted line highlights the titer of the VLB120 $\Delta 3 \mathrm{pProd}$ strain. All strains were cultivated in mineral salt medium supplemented with $25 \mathrm{mM}$ glucose and $20 \%(\mathrm{v} / \mathrm{v}) n$-decane for in-situ extraction. Methyl ketone measurements were taken after complete carbon source depletion, i.e., after $24 \mathrm{~h}$ or $48 \mathrm{~h}$. Errors represent the standard deviation of data of duplicate experiments; ${ }^{a}$ standard deviation of less than one.

\subsection{Performance of $\boldsymbol{P}$. taiwanensis VLB120 $\Delta 3$ pProd in bioreactor cultivations}

The potential of the strain VLB120 $\Delta 3$ pProd was further assessed in $\mathrm{pH}$ - and DO-controlled batch and fed-batch fermentations. Under batch conditions, $540 \mathrm{mg} \mathrm{L}^{-1}{ }^{-1}$ methyl ketones were produced with a productivity of $11 \mathrm{mg} \mathrm{L}^{-1}{ }^{-1} \mathrm{~h}^{-1}$ (Figure 3A). The dominant methyl ketones were the saturated $\mathrm{C} 11$ and $\mathrm{C} 13$ and the monounsaturated $\mathrm{C} 13: 1$ and $\mathrm{C} 15: 1$ congeners.

Significant methyl ketone production was only observed in stationary phase. The cessation of growth occurred upon nitrogen depletion, which was the limiting nutrient in the medium used while carbon was supplied in excess. Additional nitrogen supply via ammonium hydroxide titration for $\mathrm{pH}$ control was low as the strain produced only minor amounts of gluconate (Figure 3A). 
(A)

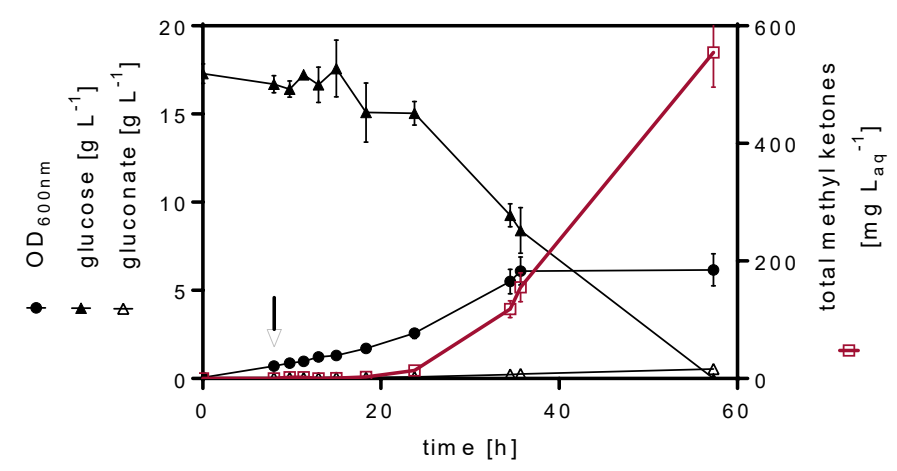

(B )

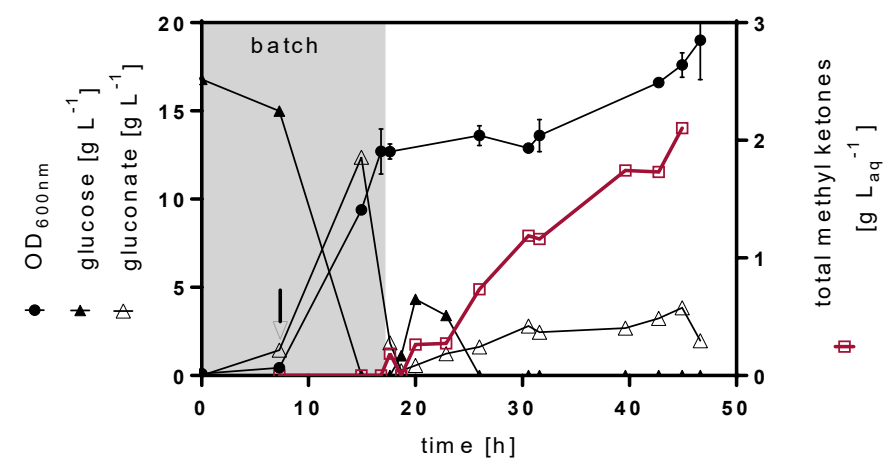

(C)

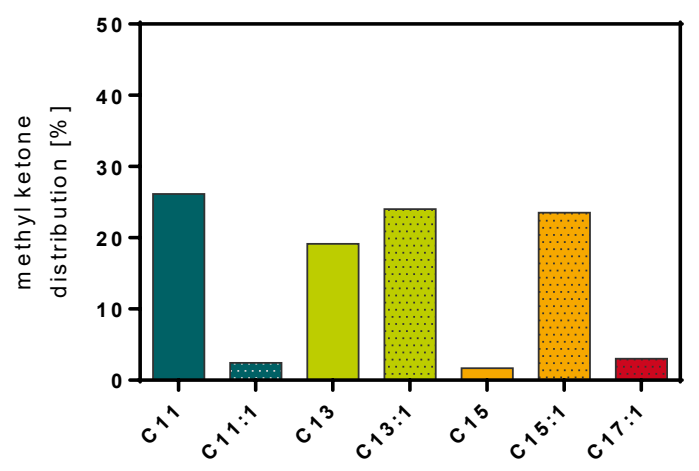

Figure 3. Performance of $P$. taiwanensis VLB120 $\Delta 3$ pProd in (A) batch and (B) fed-batch cultivation. Both cultivations were done in mineral salt medium with an initial concentration of $17 \%(v / v) n$-decane as second organic phase. The time point of induction is indicated by a vertical arrow. The total methyl ketones concentration is the sum of all detected saturated and unsaturated congeners (C11, C11:1, C13, C13:1, C15, C15:1, C17:1). (C) Methyl ketone distribution at the endpoint of the fed-batch fermentation.

To gain higher product titers, we extended the production phase by fed-batch fermentation and aimed for a similar growth limitation. Differences in growth and carbon source consumption between the initial batch experiment (Figure $3 \mathrm{~A}$ ) and the batch phase of the fed-batch cultivation (Figure $3 \mathrm{~B}$ ) are explained by an improved pre-cultivation procedure. After the end of the batch phase, glucose $(585 \mathrm{mM})$ in mineral salt medium was fed continuously at a rate of $5 \mathrm{~mL} \mathrm{~h}^{-1}$. This feed regimen results in a significant carbon excess (C:N ratio of 39:1) and potentially nitrogen-limited growth known to upregulate the fatty acid metabolism (Poblete-Castro et al., 2012b) and hence favorable for methyl ketone synthesis. During the fed-batch phase, the methyl ketone concentration increased linearly to a final titer 
of $2.1 \mathrm{~g} \mathrm{Laq}^{-1}$ (Figure 3B), corresponding to a yield of $50 \mathrm{mg} \mathrm{gGLC}^{-1}$ (Table 2). The methyl ketone distribution did not change during the feed phase (Figure $3 \mathrm{C}$ ).

\subsection{High-titer methyl ketone production after full truncation of the $\boldsymbol{\beta}$-oxidation cycle}

Further genome analysis revealed that $P$. taiwanensis VLB120 possesses three isozymes of the acetyl-CoA acyltransferase FadA encoded by fadA (PVLB_07895), PVLB_12575 (referred to as fadA2), and PVLB_26537. The gene PVLB_26537 is located on P. taiwanensis VLB120's megaplasmid, which the strain had lost during the gene knock-out procedure. We deleted the remaining fadA2 in P. taiwanensis VLB120 $\Delta 3$ to fully truncate the $\beta$-oxidation cycle and cotransformed the strain with the two production plasmids. In shake flask experiments, P. taiwanensis VLB120 $\Delta 3 \Delta$ fadA2 pProd produced $415 \mathrm{mg} \mathrm{Laq}^{-1}$ total methyl ketones, an almost 4-fold increase in methyl ketone titer (Figure 2).

\subsection{Metabolic modeling predicts genetic engineering targets for improved methyl ketone production}

To assess the potential for further advancement of methyl ketone production, we determined the theoretical maximum product yield using a metabolic model of $P$. taiwanensis VLB120 extended with the engineered methyl ketone synthesis pathway (iJN1411_MK, cf. Material and Methods section 2.7). With glucose as the sole carbon source and the experimentally determined distribution of methyl ketone congeners, the maximal theoretical yield computes to $325 \mathrm{mg} \mathrm{gGLC}$. The yield of $92 \pm 7 \mathrm{mg} \mathrm{gGLC}^{-1}$ achieved in a shake-flask cultivation of VLB120 $\Delta 3 \Delta$ fadA2 pProd (Figure 2) thus corresponds to about $30 \%$ of the maximum. The redox coenzyme demand required to achieve the maximal methyl ketone yield was computed to 89.6 mmol $_{\mathrm{NAD}(\mathrm{P}) \mathrm{H}} \mathrm{g}_{\mathrm{MK}}{ }^{-1}$, which is more than double the demand for biomass synthesis (40.6 mmol $_{\mathrm{NAD}(\mathrm{P}) \mathrm{H}} \mathrm{gCDW}^{-1}$ ) (Supplementary Figure S3). The highest reported glucose uptake rate for P. taiwanensis VLB120 of $10 \mathrm{mmol} \mathrm{gCDW}^{-1} \mathrm{~h}^{-1}$ (Rühl et al., 2009) translates into a maximal product synthesis rate of $0.6 \mathrm{~g}_{\mathrm{MK}} \mathrm{g}_{\mathrm{CDW}}{ }^{-1} \mathrm{~h}^{-1}$ and a $\mathrm{NAD}(\mathrm{P}) \mathrm{H}$ turnover of $52 \mathrm{mmol} \mathrm{gCDW}^{-1} \mathrm{~h}^{-1}$. While this is about 2-fold the rate under standard growth conditions (at a specific growth rate of $0.68 \mathrm{~h}^{-1}$ (Nies et al., 2020)), this demand does not challenge the redox cofactor regeneration

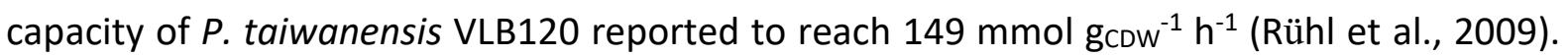
These in silico analyses indicate a substantial room for improvement of methyl ketone synthesis in $P$. taiwanensis VLB120 by metabolic engineering.

To develop VLB120 $\Delta 3 \Delta$ fadA2 pProd towards more efficient methyl ketone production, we predicted the most favorable strain designs with three to five simultaneous gene deletions using a genetic optimization algorithm and the iJN1411_MK reconstruction. The quadruple and quintuple mutants with the highest predicted fitness both showed an increase in methyl ketone yield of about 8-fold compared to the reference, which in these simulations was VLB120 $\Delta 3$ pProd. None of the triple gene deletion designs met our criteria for a minimal production enhancement of $50 \%$ and were therefore not further assessed. For intervention sets with four and five gene deletions, the genes phaC1 and tesB were present in $81 \%$ to $100 \%$ of the fittest strain designs and presented the highest observed occurrences (Figure 4). 
Since neither of these targets had been experimentally tested at this point, we decided to integrate phaC1 and tesB deletions in our engineering efforts.
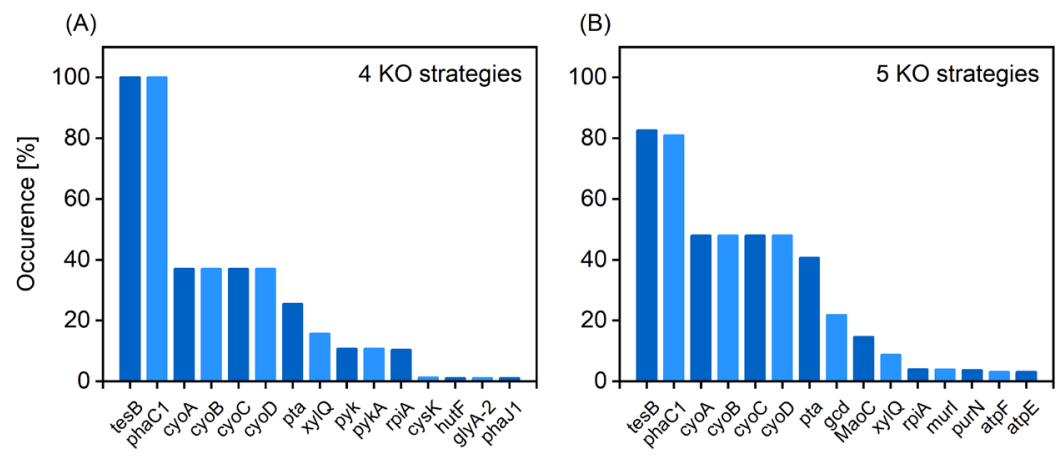

Figure 4. Occurrence distribution of gene knock-out (KO) targets in the best, unique strategies for methyl ketone overproduction determined by the genetic algorithm. (A) and (B) show results for separate genetic algorithm runs searching for quadruple and quintuple KO strategies, respectively. Only strategies that led to an increase in fitness of more than $50 \%$ compared to the reference VLB120 $\Delta 3$ pProd were considered.

\subsection{Methyl ketone production is not limited by precursor drain into polyhydroxyalkanoate synthesis}

We initially assessed the impact of individual model-predicted gene deletions in the background of strain VLB120 $\Delta 3$ pProd. We first targeted the polyhydroxyalkanoate (PHA) biosynthesis. PHAs are derived from intermediates of the de novo fatty acid synthesis and degradation (Figure 1) and are typically produced by Pseudomonas as a carbon and energy reserve under noncarbon nutrient-limiting conditions (Brandl et al., 1988). We argued that the upregulated $\beta$-oxidation cycle in the engineered Pseudomonas strain could induce PHA production under standard growth conditions, which would result in competition for precursors with methyl ketone synthesis. In P. taiwanensis VLB120, two PHA synthases are encoded in an operon together with the PHA depolymerase. We deleted the complete pha operon (PVLB_02155-PVLB_02165) but did not observe improved methyl ketone production with the resulting strain VLB120 $\Delta 3 \Delta$ pha pProd in batch shake flask experiments (Figure 2).

\subsection{Deletion of tesB boosts methyl ketone production.}

The second predicted target, tes $B$, encodes a type II thioesterase, which catalyzes the reversed reaction of the overexpressed FadD. The TesB thioesterase has a substrate specificity towards C12 to C18 (hydroxy)acyl-CoAs (Spencer et al., 1978). In previous metabolic engineering of $E$. coli, this gene was overexpressed to produce enantiopure 3-hydroxybutyrate (Tseng et al., 2009) and to reverse the flux through the $\beta$-oxidation (Dellomonaco et al., 2011). However, a knock-out of tes $B$ as a strategy to lift the synthesis of fatty acid derivatives has not been reported so far.

Deletion of tesB in VLB120 $\Delta 3$ pProd increased the total methyl ketone titer by 3-fold to 333 $\mathrm{mg} \mathrm{Laq}^{-1}$ (Figure 2). We further tested the tesB gene deletion strain VLB120 $\Delta 3 \Delta$ tesB pProd under controlled bioreactor conditions (Figure 5). Under batch conditions, VLB120 $\Delta 3 \Delta$ tesB pProd produced $1.3 \mathrm{~g} \mathrm{Laq}^{-1}$ (Figure 5A, Table 2). The increase in methyl ketone titer after carbon source depletion can be explained by a slow, spontaneous decarboxylation of the 
3-keto acid. Indeed, we had observed a significant increase in the methyl ketone titer after incubating the fermentation broth of a predecessor strain at $70^{\circ} \mathrm{C}$ (Supplementary Figure S4), supporting the hypothesis that the decarboxylation is a limiting step. However, as we observed PHA granules at the end of the batch-phase of a fed-batch fermentation of this strain (not shown), the supply of additional carbon and energy by PHA degradation cannot be excluded. The titer further increased to $6.0 \mathrm{~g} \mathrm{Laq}^{-1}$ in a fed-batch fermentation; productivity and yield also increased (Figure 5B, Table 2). The methyl ketone distribution showed no changes in comparison to the VLB120 $\Delta 3$ pProd.

(A)

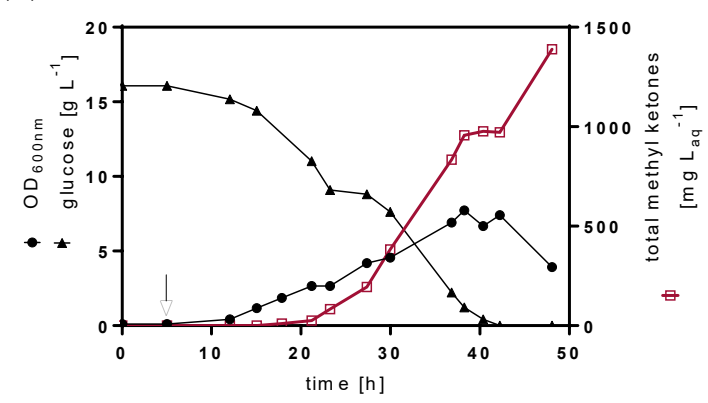

(B)

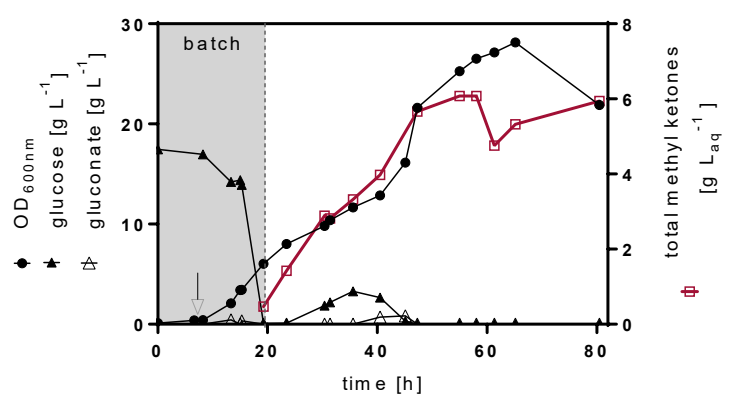

Figure 5. Methyl ketone production and growth during batch $(\mathrm{A})$ and fed-batch (B) cultivation of $P$. taiwanensis VLB120 $\Delta 3 \Delta$ tes $B$ pProd in mineral salt medium with an initial concentration of $17 \%(v / v) n$-decane as second organic phase. Shown are the time courses of the optical density and relevant substrates and products. For the reason of clarity, gluconate concentrations, which increased to low levels of $0.1 \mathrm{~g} \mathrm{~L}^{-1}$ in the batch experiment, were excluded from (A). A vertical arrow indicates the time point of induction. For both the batch and fed-batch fermentations, data of one experiment are shown.

\subsection{Consolidation of individually tested gene deletions further increases production}

The two targets predicted by metabolic modeling, tes $B$ and pha, as well as the gene fadA2 were deleted in VLB120 $\Delta 3$ pProd to test the combined effect of these three knock-outs. When the resulting strain VLB120 $\Delta 6$ pProd was cultivated without in situ product extraction into an $n$-decane overlay, we observed a white precipitate on the flask bottom after $24 \mathrm{~h}$ (Supplementary Figure S5). A GC analysis of the crystal-like precipitate dissolved in $n$-decane verified that the solids contained high amounts of methyl ketones. Only methyl ketone congeners with chain lengths between $\mathrm{C} 13$ and $\mathrm{C} 15$ were detected, while the $\mathrm{C} 11$ ketone probably did not precipitate due to the lower melting point of $15^{\circ} \mathrm{C}$ (National Center for Biotechnology Information).

In a 48-h shake flask experiment with in situ product extraction, VLB120 $\Delta 6 \mathrm{pProd}$ produced $455 \mathrm{mg} \mathrm{Laq}^{-1}$ methyl ketones, a 4-fold increase in comparison to VLB120 $\Delta 3$ pProd (Figure 2). In fed-batch fermentation, the growth of this consolidated production strain ceased after the batch phase, while the methyl ketone titer significantly increased (Figure 6). VLB120 $\Delta 6$ pProd also outperformed the predecessor strains under these cultivation conditions, in which 9.8 $\mathrm{g} \mathrm{Laq}^{-1}$ methyl ketones (corresponding to $69.3 \mathrm{~g} \mathrm{~L}$ in the $n$-decane extraction phase) were produced in less than $80 \mathrm{~h}$ (productivity of $132 \mathrm{mg} \mathrm{Laq}^{-1} \mathrm{~h}^{-1}$ ). The product yield increased to $171 \mathrm{mg} \mathrm{g}_{\mathrm{GLC}}{ }^{-1}, \sim 53 \%$ of the maximum theoretical yield (Table 2). As with the predecessor strains, C13:1, C15:1, C11, and C13 were the most dominant methyl ketone congeners. The 
fraction of unsaturated congeners was approx. $60 \%$, which is significantly higher as reported for E. coli ( $\sim 0 \%$ ) and R. eutropha ( $10 \%$ ) (Goh et al., 2012; Müller et al., 2013) but has likewise been observed for engineered $P$. putida (Dong et al., 2019). The abundance of these congeners correlates with the fraction of unsaturated fatty acid moieties in the lipids of these species, reported to be around $70 \%$ for Pseudomonas (Rühl et al., 2012), $40 \%$ for E. coli (Pramanik and Keasling, 1997) and to vary between 17-30 \% for R. eutropha (Park et al., 2011).

(A)

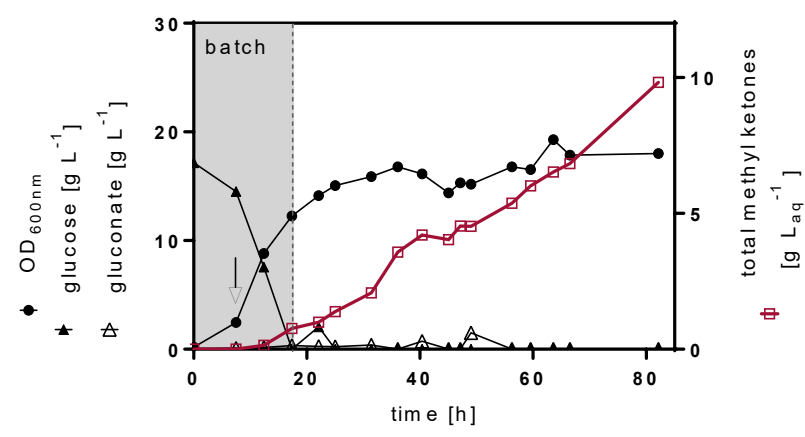

(B)

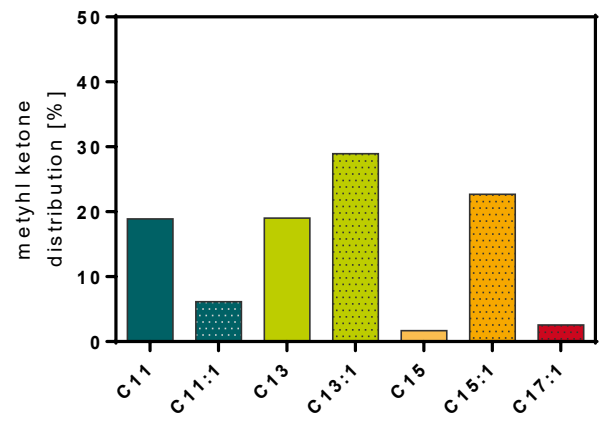

Figure 6. Methyl ketone production in P. taiwanensis VLB120 $\Delta 6$ pProd in fed-batch cultivation with an initial concentration of $17 \%(\mathrm{v} / \mathrm{v}) n$-decane as second organic phase. (A) Time course data of optical density and relevant substrates and products. Total methyl ketones represent the sum of all detected methyl ketone congeners. A vertical arrow indicates the time point of induction. (B) Methyl ketone distribution at the end of the fed-batch fermentation.

Table 2. Summary of key performance metrics of the engineered P. taiwanensis VLB120 strains in batch and fedbatch fermentations.

\begin{tabular}{l|cc|cc|c}
\hline & \multicolumn{2}{|c|}{ VLB120 $\Delta 3$ pProd } & \multicolumn{2}{c|}{ VLB120 $\Delta 3 \Delta$ tesB pProd } & \multicolumn{2}{c}{ VLB120 $\Delta 6$ pProd } \\
& batch*** & fed-batch* & batch** & fed-batch** & fed-batch* \\
\hline $\begin{array}{l}\text { total MK [g Laq } \\
\text { Productivity }\end{array}$ & $0.6 \pm 0.1$ & 2.1 & $1.3 \pm 0.2$ & $6.0 \pm 0.1$ & 9.8 \\
$\begin{array}{l}\left.\mathrm{mg} \mathrm{Laq}^{-1} \mathrm{~h}^{-1}\right] \\
\text { yield }\left[\mathrm{mg} \mathrm{gGLC}^{-1}\right]\end{array}$ & $11 \pm 1$ & 56 & $30 \pm 5$ & $82 \pm 18$ & 132 \\
\hline
\end{tabular}

${ }^{* * *} \mathrm{n}=3 ;{ }^{* *} \mathrm{n}=2{ }^{*} \mathrm{n}=1$; for $\mathrm{n}>1$, mean values and standard deviations are shown; MK, methyl ketones; GLC, glucose; aq, aqueous phase; the volumetric productivity was determined for the time period after induction of heterologous gene expression.

The proportion of unsaturated fatty acids in Pseudomonas is increased to counteract a reduction of membrane fluidity at the lower cultivation temperature of $30{ }^{\circ} \mathrm{C}$ (Demendoza and Cronan, 1983; Loffhagen et al., 2004). Accordingly, higher amounts of monounsaturated methyl ketones were observed in $E$. coli when grown at a reduced temperature supporting this relationship (Goh et al., 2012). For the utilization of methyl ketones as biofuel, this elevated fraction of monounsaturated methyl ketones is disadvantageous. While unsaturation can reduce the melting point, it also negatively affects the fuel properties regarding the cetane number (Knothe, 2008). We determined a derived cetane number (DCN) of 54 for the methyl ketone mixture produced by $P$. taiwanensis VLB120 (Supplementary Table S2). Although still in the diesel range, this value is much lower compared to the DCN of approx. 63 calculated for a mixture of fully saturated methyl ketone congeners with the same 
chain length distribution. This disadvantage of the Pseudomonas hosts could be addressed by engineering the specificity of acyl-CoA thioesterases towards saturated fatty acids (Hernandez Lozada et al., 2018) or modulating the fatty acid composition by adapting Pseudomonas to growth at higher temperatures.

\section{Conclusion}

By applying rational and model-guided metabolic engineering, P. taiwanensis VLB120 was successfully developed for medium-chain length methyl ketones production at high titer and yield surpassing values achieved with alternative hosts. Notably, this high performance was achieved solely by directing the carbon flux through de novo fatty acid synthesis towards methyl ketone synthesis. Key genetic modifications necessary for the upregulated $\beta$-oxidation to be fully effective were the complete disruption of the $\beta$-oxidation cycle and the reverse flux from fatty acyl-CoA esters to free fatty acids. Optimization of the redox metabolism, such as redox cofactor engineering, shown to be important for methyl ketone production in E. coli, was not pursued. We argue that the high flexibility of Pseudomonas to balance redox cofactors (Kohlstedt and Wittmann, 2019; Nies et al., 2020; Nikel et al., 2016) renders such elaborate engineering efforts superfluous. Moreover, the performed in silico analysis showed that the capacity of the strain for methyl ketone production has not been fully exhausted and can be increased further by metabolic engineering. The early evaluation of cultivation strategies also showed the impact of the fermentation settings on the strain performance with nitrogen-limited, carbon-excess conditions benefitting a high flux towards methyl ketone synthesis. We believe that the full potential of $P$. taiwanensis VLB120 remained untapped in the initial, unoptimized fed-batch experiments as the rather low glucose feed rate prevented utilization of $P$. taiwanensis VLB120's high redox cofactor regeneration capacity for maximal methyl ketone synthesis. Future fermentation process optimization will further leverage the strain's metabolic capability for redox cofactor supply and conversion of non-edible renewable resources or waste streams such as lignocellulosic biomass or glycerol.

\section{Acknowledgment}

We are grateful to Andrea König (AVT, RWTH Aachen University, DE) for the prediction of fuel properties.

\section{Funding}

This study was conducted within the ERA SynBio project SynPath (Grant ID 031A459) with the financial support of the German Federal Ministry of Education and Research and was part of the DOE Joint BioEnergy Institute (https://www.jbei.org) supported by the U.S. Department of Energy, Office of Science, Office of Biological and Environmental Research through contract DE-AC02-05CH11231 between Lawrence Berkeley National Laboratory and the U.S. Department of Energy. BEE acknowledges support by the UQ-CSIRO Synthetic Biology Alliance. The laboratory of LMB is partially funded by the Deutsche Forschungsgemeinschaft 
(DFG, German Research Foundation) under Germany's Excellence Strategy within the Cluster of Excellence EXC 2186 'The Fuel Science Center'. The GC-MS/MS was also funded by the DFG under grant agreement No. 233069590. ANTP has received funding from the European Union's Horizon 2020 research and innovation program under the Marie Sklodowska-Curie grant agreement No. 793158. The funders had no role in the study design; in the collection, analysis, and interpretation of data; in the writing of the report; and in the decision to submit the article for publication.

Declarations of interest: JDK has a financial interest in Amyris, Lygos, Demetrix, Napigen, Maple Bio, Apertor Labs, Ansa Biotechnologies, and Berkeley Brewing Sciences.

\section{References}

Alter, T. B., Blank, L. M., Ebert, B. E., 2018. Genetic optimization algorithm for metabolic engineering revisited. Metabolites. 8, 33.

Blank, L. M., Ionidis, G., Ebert, B. E., Buhler, B., Schmid, A., 2008. Metabolic response of Pseudomonas putida during redox biocatalysis in the presence of a second octanol phase. FEBS J. 275, 5173-5190.

Brandl, H., Gross, R. A., Lenz, R. W., Fuller, R. C., 1988. Pseudomonas oleovorans as a source of poly ( $\beta$-hydroxyalkanoates) for potential applications as biodegradable polyesters. Appl. Environ. Microbiol. 54, 1977-1982.

Brochado, A. R., Andrejev, S., Maranas, C. D., Patil, K. R., 2012. Impact of stoichiometry representation on simulation of genotype-phenotype relationships in metabolic networks. PLoS Comput. Biol. 8, e1002758.

Buist, P. H., 2010. Unsaturated fatty acids. In: Liu, H.-W., Mander, L., Eds.), Comprehensive Natural Products II. Elsevier, Oxford, pp. 5-33.

Choi, K. H., Kumar, A., Schweizer, H. P., 2006. A 10-min method for preparation of highly electrocompetent Pseudomonas aeruginosa cells: application for DNA fragment transfer between chromosomes and plasmid transformation. J. Microbiol. Methods. 64, 391-397.

Chomczynski, P., Rymaszewski, M., 2006. Alkaline polyethylene glycol-based method for direct PCR from bacteria, eukaryotic tissue samples, and whole blood. BioTechniques. 40, 454, 456, 458.

Dahmen, M., Marquardt, W., 2015. A novel group contribution method for the prediction of the derived cetane number of oxygenated hydrocarbons. Energy \& Fuels. 29, 57815801.

Dahmen, M., Marquardt, W., 2017. Model-based formulation of biofuel blends by simultaneous product and pathway design. Energy \& Fuels. 31, 4096-4121.

Davis, M. S., Solbiati, J., Cronan, J. E., Jr., 2000. Overproduction of acetyl-CoA carboxylase activity increases the rate of fatty acid biosynthesis in Escherichia coli. J. Biol. Chem. 275, 28593-28598.

Dellomonaco, C., Clomburg, J. M., Miller, E. N., Gonzalez, R., 2011. Engineered reversal of the $\beta$-oxidation cycle for the synthesis of fuels and chemicals. Nature. 476, 355-359.

Demendoza, D., Cronan, J. E., 1983. Thermal regulation of membrane lipid fluidity in bacteria. Trends Biochem. Sci. 8, 49-52. 
Ditta, G., Stanfield, S., Corbin, D., Helinski, D. R., 1980. Broad host range DNA cloning system for gram-negative bacteria: construction of a gene bank of Rhizobium meliloti. Proc Natl Acad Sci U S A. 77, 7347-7351.

Dong, J., Chen, Y., Benites, V. T., Baidoo, E. E. K., Petzold, C. J., Beller, H. R., Eudes, A., Scheller, H. V., Adams, P. D., Mukhopadhyay, A., Simmons, B. A., Singer, S. W., 2019. Methyl ketone production by Pseudomonas putida is enhanced by plant-derived amino acids. Biotechnol. Bioeng. 116, 1909-1922.

Ebert, B. E., Kurth, F., Grund, M., Blank, L. M., Schmid, A., 2011. Response of Pseudomonas putida KT2440 to increased NADH and ATP demand. Appl. Environ. Microbiol. 77, 6597-6605.

Goh, E. B., Baidoo, E. E., Keasling, J. D., Beller, H. R., 2012. Engineering of bacterial methyl ketone synthesis for biofuels. Appl. Environ. Microbiol. 78, 70-80.

Goh, E. B., Baidoo, E. E. K., Burd, H., Lee, T. S., Keasling, J. D., Beller, H. R., 2014. Substantial improvements in methyl ketone production in E. coli and insights on the pathway from in vitro studies. Metab. Eng. 26, 67-76.

Goh, E. B., Chen, Y., Petzold, C. J., Keasling, J. D., Beller, H. R., 2018. Improving methyl ketone production in Escherichia coli by heterologous expression of NADH-dependent FabG. Biotechnol. Bioeng. 115, 1161-1172.

Hanahan, D., 1985. Techniques for transformation of E. coli. IRL Press, Oxford.

Hanko, E. K. R., Denby, C. M., Sanchez, I. N. V., Lin, W., Ramirez, K. J., Singer, C. A., Beckham, G. T., Keasling, J. D., 2018. Engineering $\beta$-oxidation in Yarrowia lipolytica for methyl ketone production. Metab. Eng. 48, 52-62.

Harrison, K. W., Harvey, B. G., 2018. High cetane renewable diesel fuels prepared from biobased methyl ketones and diols. Sustain Energ Fuels. 2, 367-371.

Hartmans, S., Smits, J. P., van der Werf, M. J., Volkering, F., de Bont, J. A., 1989. Metabolism of Styrene Oxide and 2-Phenylethanol in the Styrene-Degrading Xanthobacter Strain 124X. Appl. Environ. Microbiol. 55, 2850-2855.

Hernandez Lozada, N. J., Lai, R. Y., Simmons, T. R., Thomas, K. A., Chowdhury, R., Maranas, C. D., Pfleger, B. F., 2018. Highly active C8-acyl-ACP thioesterase variant isolated by a synthetic selection strategy. ACS Synth Biol. 7, 2205-2215.

Janssen, H. J., Steinbüchel, A., 2014. Fatty acid synthesis in Escherichia coli and its applications towards the production of fatty acid based biofuels. Biotechnol Biofuels. 7, 7.

Kim, H. M., Chae, T. U., Choi, S. Y., Kim, W. J., Lee, S. Y., 2019. Engineering of an oleaginous bacterium for the production of fatty acids and fuels. Nat. Chem. Biol. 15, 721-729.

Kimps, N. W., Bissinger, B. W., Apperson, C. S., Sonenshine, D. E., Roe, R. M., 2011. First report of the repellency of 2-tridecanone against ticks. Med. Vet. Entomol. 25, 202-208.

Knop, V., Loos, M., Pera, C., Jeuland, N., 2014. A linear-by-mole blending rule for octane numbers of $n$-heptane/iso-octane/toluene mixtures. Fuel. 115, 666-673.

Knothe, G., 2008. "Designer" biodiesel: optimizing fatty ester composition to improve fuel properties. Energy \& Fuels. 22, 1358-1364.

Kohler, K. A., Blank, L. M., Frick, O., Schmid, A., 2015. D-Xylose assimilation via the Weimberg pathway by solvent-tolerant Pseudomonas taiwanensis VLB120. Environ. Microbiol. 17, 156-170.

Kohler, K. A., Ruckert, C., Schatschneider, S., Vorholter, F. J., Szczepanowski, R., Blank, L. M., Niehaus, K., Goesmann, A., Puhler, A., Kalinowski, J., Schmid, A., 2013. Complete genome sequence of Pseudomonas sp. strain VLB120 a solvent tolerant, styrene degrading bacterium, isolated from forest soil. J. Biotechnol. 168, 729-730. 
Kohlstedt, M., Wittmann, C., 2019. GC-MS-based ${ }^{13} \mathrm{C}$ metabolic flux analysis resolves the parallel and cyclic glucose metabolism of Pseudomonas putida KT2440 and Pseudomonas aeruginosa PAO1. Metab. Eng. 54, 35-53.

Lee, H. C., Kim, J. S., Jang, W., Kim, S. Y., 2009. Thymidine production by overexpressing NAD ${ }^{+}$ kinase in an Escherichia coli recombinant strain. Biotechnol. Lett. 31, 1929-1936.

Lennen, R. M., Pfleger, B. F., 2013. Microbial production of fatty acid-derived fuels and chemicals. Curr. Opin. Biotechnol. 24, 1044-1053.

Lewis, N. E., Hixson, K. K., Conrad, T. M., Lerman, J. A., Charusanti, P., Polpitiya, A. D., Adkins, J. N., Schramm, G., Purvine, S. O., Lopez-Ferrer, D., Weitz, K. K., Eils, R., Konig, R., Smith, R. D., Palsson, B. O., 2010. Omic data from evolved E. coli are consistent with computed optimal growth from genome-scale models. Mol. Syst. Biol. 6, 390.

Loffhagen, N., Hartig, C., Babel, W., 2004. Pseudomonas putida NCTC 10936 balances membrane fluidity in response to physical and chemical stress by changing the saturation degree and the trans/cis ratio of fatty acids. Biosci Biotechnol Biochem. 68, 317-323.

Longo, M. A. S., M.A. , 2006. Production of food aroma compounds: microbial and enzymatic methodologies. Food Technol. Biotechnol. 44, 335-353.

López-Lara, I. M., Geiger, O., 2010. Formation of fatty acids. In: Timmis, K. N., (Ed.), Handbook of Hydrocarbon and Lipid Microbiology. Springer Berlin Heidelberg, Berlin, Heidelberg, pp. 385-393.

Martínez-García, E., de Lorenzo, V., 2011. Engineering multiple genomic deletions in Gramnegative bacteria: analysis of the multi-resistant antibiotic profile of Pseudomonas putida KT2440. Environ. Microbiol. 13, 2702-2716.

Müller, J., MacEachran, D., Burd, H., Sathitsuksanoh, N., Bi, C., Yeh, Y. C., Lee, T. S., Hillson, N. J., Chhabra, S. R., Singer, S. W., Beller, H. R., 2013. Engineering of Ralstonia eutropha $\mathrm{H} 16$ for autotrophic and heterotrophic production of methyl ketones. Appl. Environ. Microbiol. 79, 4433-4439.

National Center for Biotechnology Information, P. D., 2-Undecanone, CID=8163, https://pubchem.ncbi.nlm.nih.gov/compound/2-Undecanone.

Nie, L., Ren, Y., Schulz, H., 2008. Identification and characterization of Escherichia coli thioesterase III that functions in fatty acid $\beta$-oxidation. Biochemistry. 47, 7744-7751.

Nies, S. C., Dinger, R., Chen, Y., Wordofa, G. G., Kristensen, M., Schneider, K., Buchs, J., Petzold, C. J., Keasling, J. D., Blank, L. M., Ebert, B. E., 2020. A systems analysis of NADH dehydrogenase mutants reveals flexibility and limits of Pseudomonas taiwanensis VLB120's metabolism. Appl. Environ. Microbiol.

Nijkamp, K., van Luijk, N., de Bont, J. A., Wery, J., 2005. The solvent-tolerant Pseudomonas putida S12 as host for the production of cinnamic acid from glucose. Appl. Microbiol. Biotechnol. 69, 170-177.

Nikel, P. I., Perez-Pantoja, D., de Lorenzo, V., 2016. Pyridine nucleotide transhydrogenases enable redox balance of Pseudomonas putida during biodegradation of aromatic compounds. Environ. Microbiol. 18, 3565-3582.

Nogales, J., Gudmundsson, S., Duque, E., Ramos, J. L., Palsson, B. O., 2017. Expanding the computable reactome in Pseudomonas putida reveals metabolic cycles providing robustness. bioRxiv. 139121.

Panke, S., Witholt, B., Schmid, A., Wubbolts, M. G., 1998. Towards a biocatalyst for (S)-styrene oxide production: characterization of the styrene degradation pathway of Pseudomonas sp. strain VLB120. Appl. Environ. Microbiol. 64, 2032-2043. 
Park, J., Rodriguez-Moya, M., Li, M., Pichersky, E., San, K. Y., Gonzalez, R., 2012. Synthesis of methyl ketones by metabolically engineered Escherichia coli. J. Ind. Microbiol. Biotechnol. 39, 1703-1712.

Park, J. M., Kim, T. Y., Lee, S. Y., 2011. Genome-scale reconstruction and in silico analysis of the Ralstonia eutropha $\mathrm{H} 16$ for polyhydroxyalkanoate synthesis, lithoautotrophic growth, and 2-methyl citric acid production. BMC Syst Biol. 5, 101.

Poblete-Castro, I., Becker, J., Dohnt, K., dos Santos, V. M., Wittmann, C., 2012a. Industrial biotechnology of Pseudomonas putida and related species. Appl. Microbiol. Biotechnol. 93, 2279-2290.

Poblete-Castro, I., Escapa, I. F., Jager, C., Puchalka, J., Lam, C. M., Schomburg, D., Prieto, M. A., Martins dos Santos, V. A., 2012b. The metabolic response of P. putida KT2442 producing high levels of polyhydroxyalkanoate under single- and multiple-nutrientlimited growth: highlights from a multi-level omics approach. Microb Cell Fact. 11, 34.

Pramanik, J., Keasling, J. D., 1997. Stoichiometric model of Escherichia coli metabolism: incorporation of growth-rate dependent biomass composition and mechanistic energy requirements. Biotechnol. Bioeng. 56, 398-421.

Rühl, J., Hein, E. M., Hayen, H., Schmid, A., Blank, L. M., 2012. The glycerophospholipid inventory of Pseudomonas putida is conserved between strains and enables growth condition-related alterations. Microb. Biotechnol. 5, 45-58.

Rühl, J., Schmid, A., Blank, L. M., 2009. Selected Pseudomonas putida strains able to grow in the presence of high butanol concentrations. Appl. Environ. Microbiol. 75, 4653-4656.

Sambrook, J., E.F. Fritsch, and T. Maniatis, 1982. Molecular cloning: A laboratory manual. Cold Spring Harbour Press.

Sanchez, A. M., Andrews, J., Hussein, I., Bennett, G. N., San, K. Y., 2006. Effect of overexpression of a soluble pyridine nucleotide transhydrogenase (UdhA) on the production of poly(3-hydroxybutyrate) in Escherichia coli. Biotechnol. Prog. 22, 420425.

Spencer, A. K., Greenspan, A. D., Cronan, J. E., Jr., 1978. Thioesterases I and II of Escherichia coli. Hydrolysis of native acyl-acyl carrier protein thioesters. J. Biol. Chem. 253, 59225926.

Steen, E. J., Kang, Y., Bokinsky, G., Hu, Z., Schirmer, A., McClure, A., Del Cardayre, S. B., Keasling, J. D., 2010. Microbial production of fatty-acid-derived fuels and chemicals from plant biomass. Nature. 463, 559-562.

Tee, T. W., Chowdhury, A., Maranas, C. D., Shanks, J. V., 2014. Systems metabolic engineering design: fatty acid production as an emerging case study. Biotechnol. Bioeng. 111, 849857.

Tseng, H. C., Martin, C. H., Nielsen, D. R., Prather, K. L., 2009. Metabolic engineering of Escherichia coli for enhanced production of $(R)$ - and (S)-3-hydroxybutyrate. Appl. Environ. Microbiol. 75, 3137-3145.

Verhoef, S., Ballerstedt, H., Volkers, R. J., de Winde, J. H., Ruijssenaars, H. J., 2010. Comparative transcriptomics and proteomics of $\mathrm{p}$-hydroxybenzoate producing Pseudomonas putida S12: novel responses and implications for strain improvement. Appl. Microbiol. Biotechnol. 87, 679-690.

Wierckx, N. J., Ballerstedt, H., de Bont, J. A., Wery, J., 2005. Engineering of solvent-tolerant Pseudomonas putida S12 for bioproduction of phenol from glucose. Appl. Environ. Microbiol. 71, 8221-8227. 
Wynands, B., Lenzen, C., Otto, M., Koch, F., Blank, L. M., Wierckx, N., 2018. Metabolic engineering of Pseudomonas taiwanensis VLB120 with minimal genomic modifications for high-yield phenol production. Metab. Eng. 47, 121-133.

Xu, P., Qiao, K., Ahn, W. S., Stephanopoulos, G., 2016. Engineering Yarrowia lipolytica as a platform for synthesis of drop-in transportation fuels and oleochemicals. Proc Natl Acad Sci U S A. 113, 10848-10853.

Zobel, S., Benedetti, I., Eisenbach, L., de Lorenzo, V., Wierckx, N., Blank, L. M., 2015. Tn7-Based Device for Calibrated Heterologous Gene Expression in Pseudomonas putida. ACS Synth Biol. 4, 1341-1351. 


\section{Supplementary Material}

A

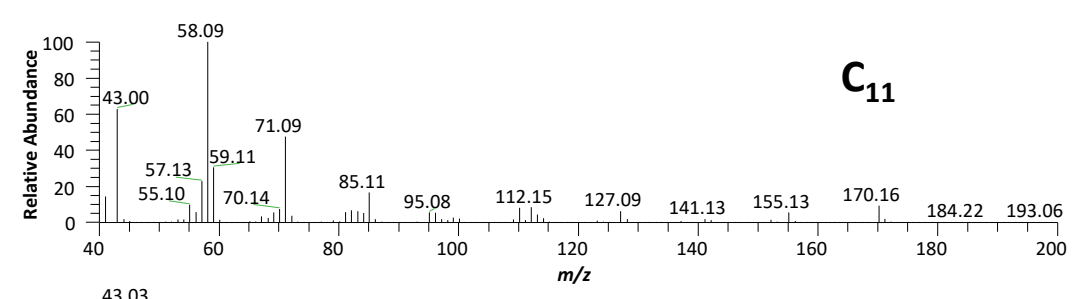

B

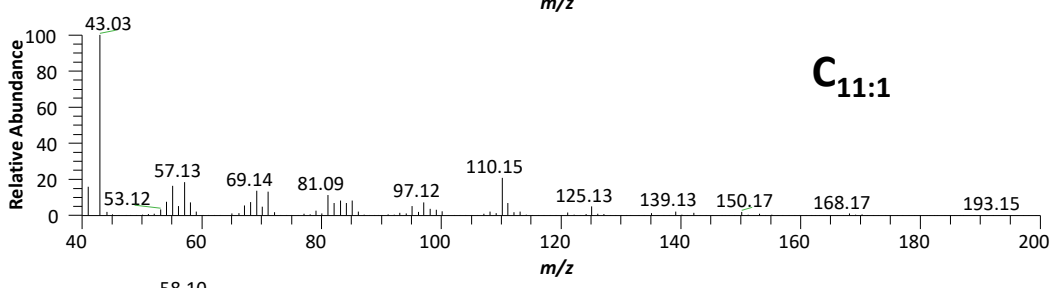

C

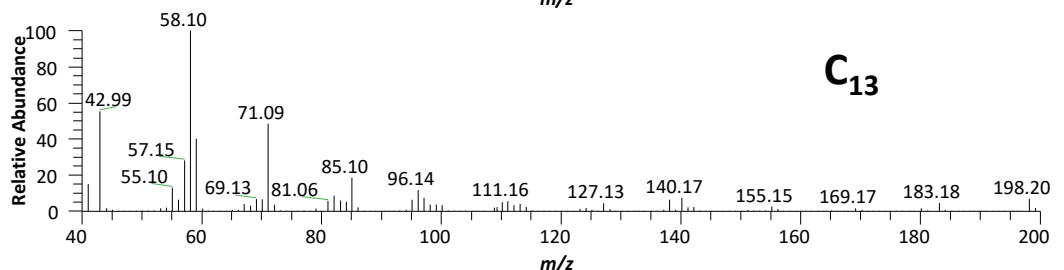

D

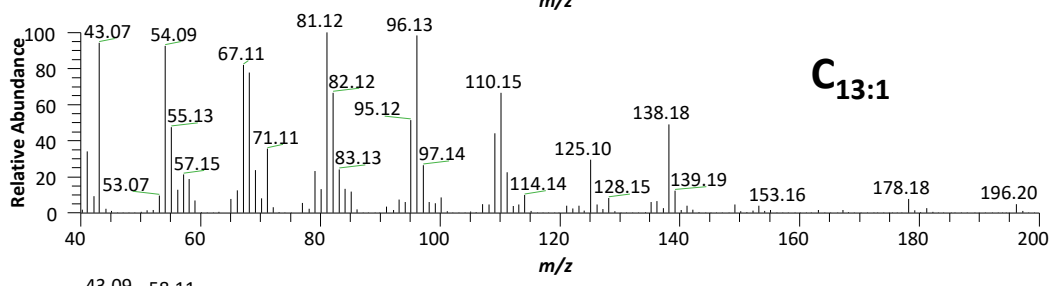

E

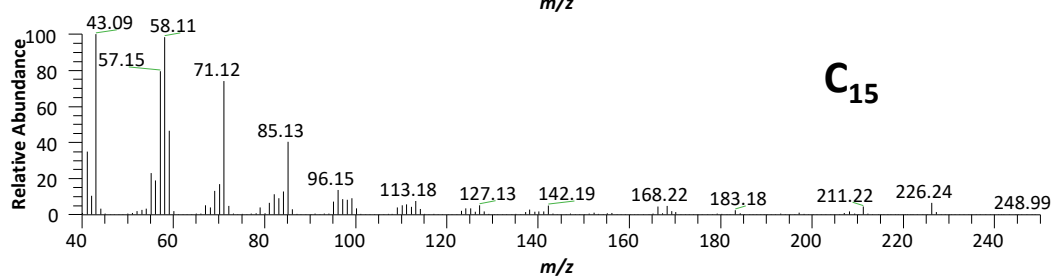

$\mathbf{F}$

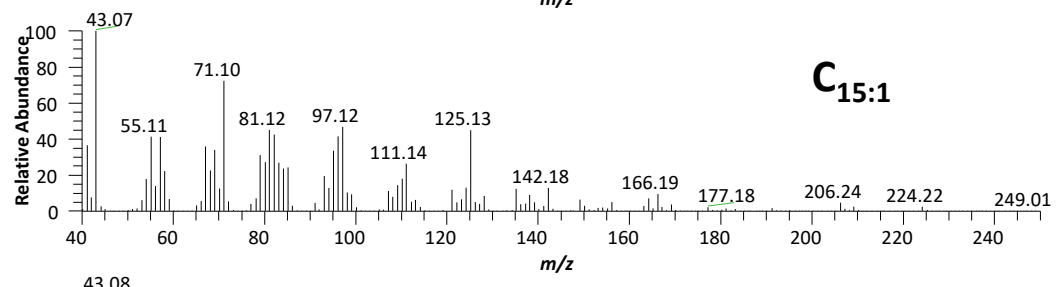

G

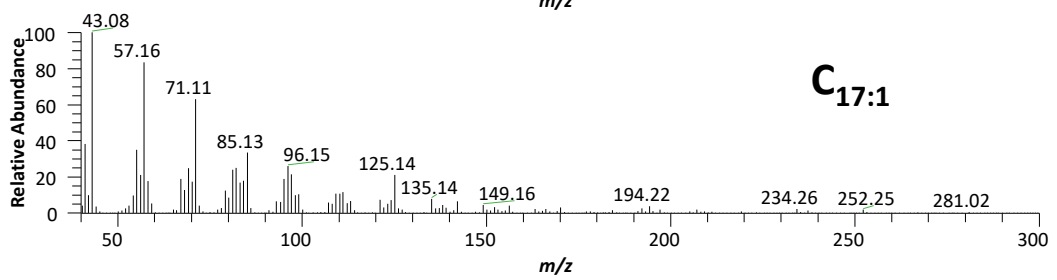

Supplementary Figure S1. Mass spectra of methyl ketones generated by VLB120 $\Delta 6$ pProd at 66 h. (A) $C_{11}$, (B)

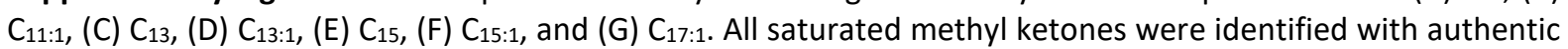
standards. The monounsaturated methyl ketones were tentatively identified by comparison with spectra shown in (Goh et al., 2012). 

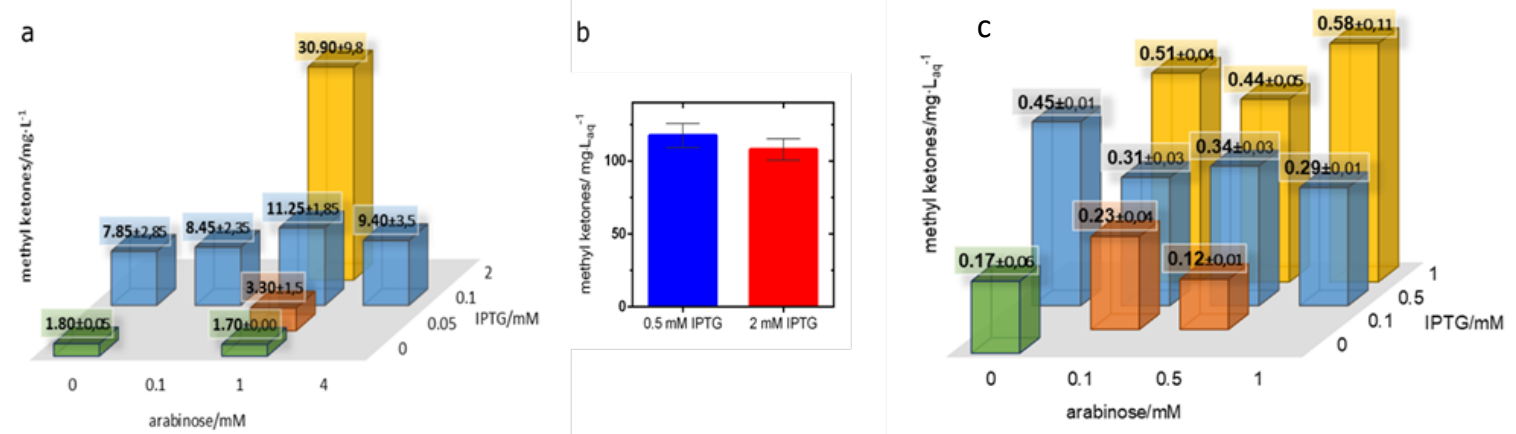

Supplementary Figure S2. Determination of optimal inducer concentrations for methyl ketone production. The inducers IPTG and arabinose were added to the shake flask cultures $4.5 \mathrm{~h}$ after inoculation. The methyl ketone titers shown are the mean of duplicate experiments with the corresponding standard deviation and were measured after $24 \mathrm{~h}$ of cultivation. a) VLB120 $\Delta 3$ pProd was grown in $100 \mathrm{~mL}$ shake flasks without $n$-decane overlay. The overall titer of detected methyl ketone congeners (C11, C13, C15) is shown. b) VLB120 $\Delta 3$ pProd was grown in $250 \mathrm{~mL}$ shake flasks with $n$-decane overlay and induced with $1 \mathrm{mM}$ arabinose and either 0.5 or 2 mM IPTG. The overall titer of detected methyl ketone congeners (C11, C13, C13:1, C15, C15:1) related to the aqueous phase is shown. c) VLB120 $\Delta 3$ attTn7::MK was grown in $100 \mathrm{~mL}$ shake flasks with a $n$-decane overlay. The overall titer of detected methyl ketones congeners $(C 11, C 13, C 13: 1, C 15, C 15: 1)$ related to the aqueous phase is shown.

(A)

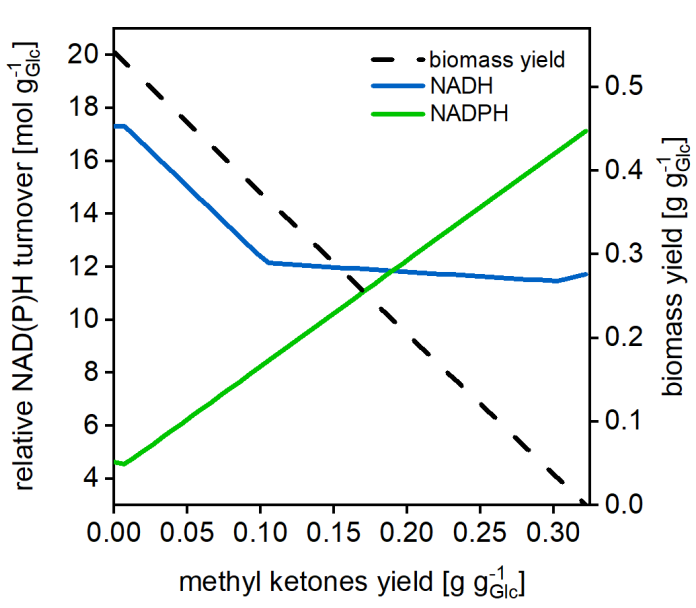

(B)

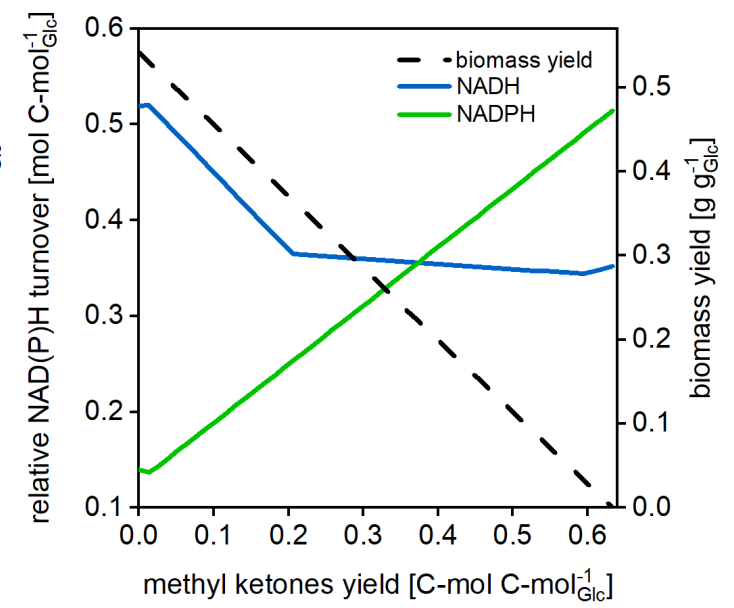

Supplementary Figure S3. Simulated $\mathrm{NAD}(\mathrm{P}) \mathrm{H}$ turnover and biomass yield as a function of a given methyl ketone yield. (A) mass related, $(B)$ on $C$-mol basis. The $N A D(P) H$ turnover values and biomass yields were derived from growth-optimal flux distributions at a fixed methyl ketone production rates. Biomass yields were computed by dividing the growth rate by the glucose uptake rate. The $N A D(P) H$ turnover depicts the sum of fluxes of all reactions that produce $\mathrm{NAD}(\mathrm{P}) \mathrm{H}$ divided by the glucose uptake rate. All $\mathrm{NAD}(\mathrm{P}) \mathrm{H}$ producing fluxes were multiplied with the stoichiometric coefficient of $\mathrm{NAD}(\mathrm{P}) \mathrm{H}$ for the respective reaction. Note that for constraintbased modeling approaches, as applied here, metabolite concentrations are assumed to be constant. Thus, summation of all $\mathrm{NAD}(\mathrm{P}) \mathrm{H}$ consuming fluxes yields the same $\mathrm{NAD}(\mathrm{P}) \mathrm{H}$ turnover values. The iJN1411 model, including the methyl ketone production pathway, was used for all simulations. The methyl ketone distribution was set as depicted by the experimental results for VLB120 $\Delta 3$ pProd (cf. Results, section 3.3). 


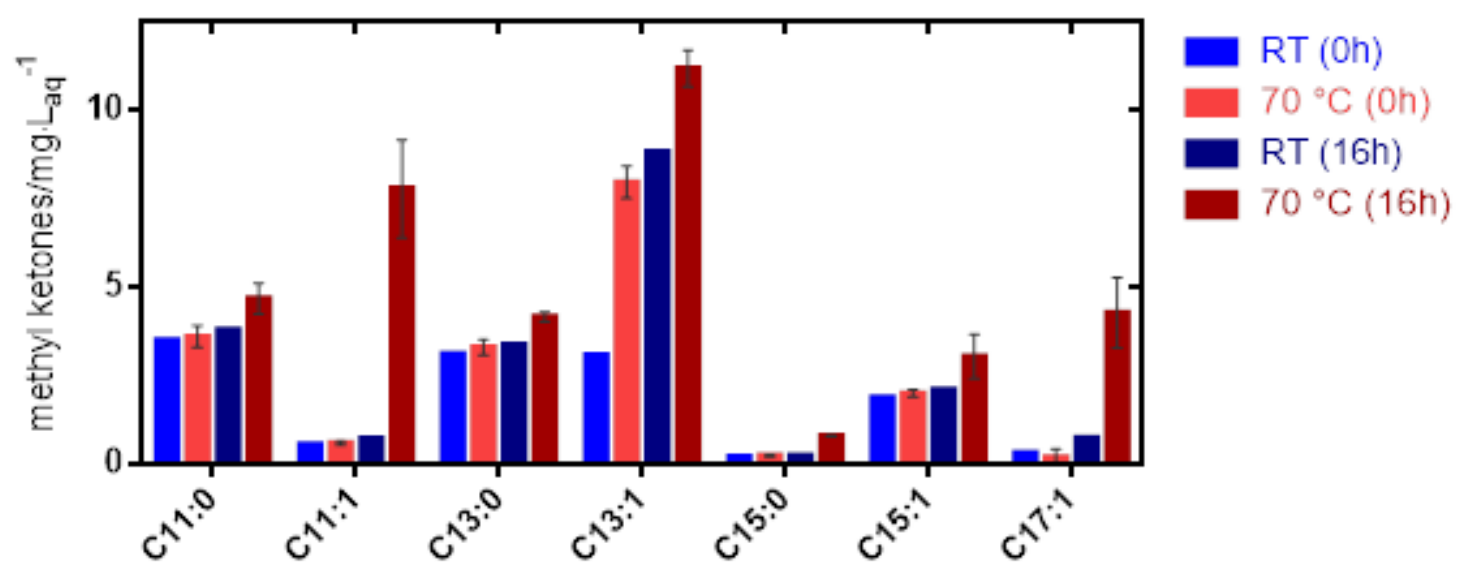

Supplementary Figure S4. Enhanced decarboxylation of 3-ketoacids by incubation of culture broth at $70{ }^{\circ} \mathrm{C}$ for $16 \mathrm{~h}$ increases methyl ketones titers. Three samples from a culture of VLB120 $\Delta 3$ pProd were taken; two were incubated at $70{ }^{\circ} \mathrm{C}$ while the third sample was left at room temperature (RT). The concentrations of saturated $(\mathrm{C} 11, \mathrm{C} 13, \mathrm{C} 15)$ and monounsaturated $(\mathrm{C} 11: 1, \mathrm{C} 13: 1, \mathrm{C} 15: 1, \mathrm{C} 17: 1)$ methyl ketone congeners were determined before $(0 \mathrm{~h})$ and after the incubation period (16h). Error bars represent the standard deviation of the duplicate experiment at $70^{\circ} \mathrm{C}$.

\section{(A)}

(B)

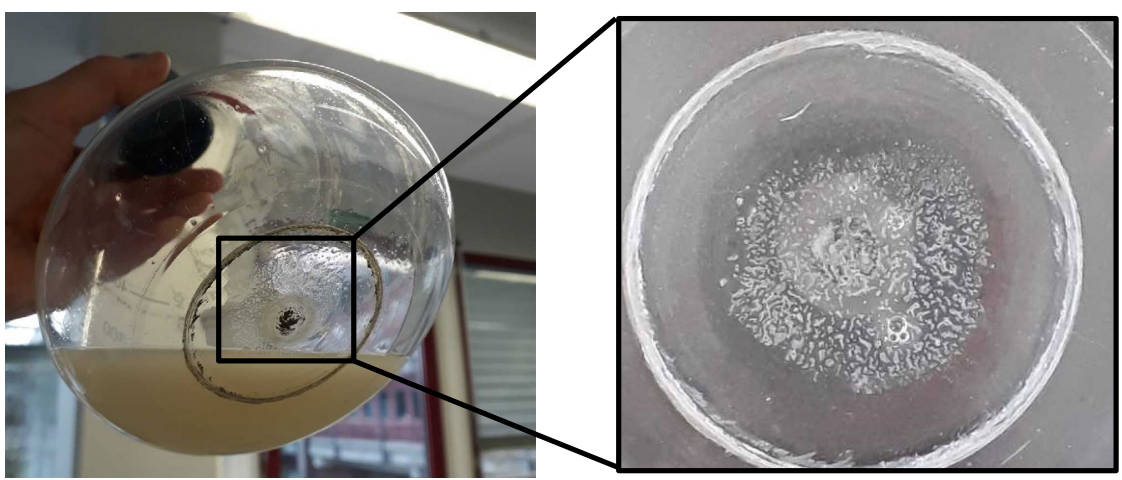

Supplementary Figure S5. Cultivation of $P$. taiwanensis VLB120 $\Delta 6$ pProd in mineral salt medium without $n$ decane as an organic phase. White crystal-like precipitation on the flask bottom after $24 \mathrm{~h}$ cultivation. (B) View of the flask bottom from above. 
Supplementary Table S1. Primers used in this study

\begin{tabular}{|c|c|c|}
\hline Primer & Sequence $\left(5^{\prime}-3^{\prime}\right)^{a}$ & Description \\
\hline \multicolumn{3}{|c|}{ Gene deletions } \\
\hline SN075 & gataacagggtaatctgGGACCGCATGAAAGATGACC & TS1 fadA (PVLB_07895) forward \\
\hline SN076 & cactcaccCTCGCTCTACTCTTGCTCTC & TS1 fadA (PVLB_07895) reverse \\
\hline SN077 & agagcgagGGTGAGTGACATGCAGATAC & TS2 fadA (PVLB_07895) forward \\
\hline SN078 & cgggtaccgagctcgGTCTCGGATATGGCCGATAC & TS2 fadA (PVLB_07895) reverse \\
\hline SN091 & GCGCATCGACAAGGTCATGG & $\begin{array}{l}\text { Verification cointegration fadA } \\
\text { forward }\end{array}$ \\
\hline SN092 & AGGCAGGATCTCGTACTTGG & $\begin{array}{l}\text { Verification cointegration fadA } \\
\text { reverse }\end{array}$ \\
\hline SN093 & agggataacagggtaatctgCGGACGATGGCGTTGGATTC & TS1 fadE (PVLB_07056) forward \\
\hline SN094 & ttgcaaggtgGCTTGAGCCGTCCCAGATAG & TS1 fadE (PVLB_07056) reverse \\
\hline SN095 & cggctcaagcCACCTTGCAATCACCGATAC & TS2 fadE (PVLB_07056) forward \\
\hline SN096 & atccccgggtaccgagctcgCGGTCCTTCAACAGGTGATG & TS2 fadE (PVLB_07056) reverse \\
\hline SN089 & TGGTCCAGTCCATCCATTTG & $\begin{array}{l}\text { Verification cointegration fadE } \\
\text { forward }\end{array}$ \\
\hline SN090 & GCCTGTCTGCCCATGATGTG & $\begin{array}{l}\text { Verification cointegration fadE } \\
\text { reverse }\end{array}$ \\
\hline SN083 & gataacagggtaatctgAACAACGGCGGCAGGATCAC & TS1 fadE2 (PVLB_10075) forward \\
\hline SN084 & tcagcacaCATGGACCAGAGCCTAGAAC & TS1 fadE2 (PVLB_10075) reverse \\
\hline SN085 & ggtccatgTGTGCTGATCACGCAGTTTC & TS2 fadE2 (PVLB_10075) forward \\
\hline SN086 & cgggtaccgagctcgTGGCGTGGGCCAGTTCAAAG & TS2 fadE2 (PVLB_10075) reverse \\
\hline SN087 & CGGCGAAATCCAGTCTACCG & $\begin{array}{l}\text { Verification cointegration fadE2 } \\
\text { forward }\end{array}$ \\
\hline SN088 & ATCTTCGCTTCCAGGGTATC & $\begin{array}{l}\text { Verification cointegration fadE2 } \\
\text { reverse }\end{array}$ \\
\hline SN041 & gataacagggtaatctgGGCATGACAGCGGTTTGTTC & $\begin{array}{l}\text { TS1 pha (PVLB_02155-02165) } \\
\text { forward }\end{array}$ \\
\hline SN042 & tcaaggtgGTTGTCCTGAGACGCGTACC & $\begin{array}{l}\text { TS1 pha (PVLB_02155-02165) } \\
\text { reverse }\end{array}$ \\
\hline SN043 & aggacaacCACCTTGACCGGATGAAGAC & $\begin{array}{l}\text { TS2 pha (PVLB_02155-02165) } \\
\text { forward }\end{array}$ \\
\hline SN044 & cgggtaccgagctcgCCGCTGGTAATCCAGCGAAC & $\begin{array}{l}\text { TS2 pha (PVLB_02155-02165) } \\
\text { reverse }\end{array}$ \\
\hline SN065 & ACGTCGGTCTGGTCGGTTTG & $\begin{array}{l}\text { Verification cointegration PHA } \\
\text { forward }\end{array}$ \\
\hline SN066 & AGCATCATCACCTGGTAGAC & $\begin{array}{l}\text { Verification cointegration PHA } \\
\text { reverse }\end{array}$ \\
\hline SN283 & agggataacagggtaatctgCCGCGCCTACGCTTTAAAC & TS1 tesB (PVLB_03305) forward \\
\hline SN284 & tcctgggtggGCAAGGCATCACAGCTCTTC & TS1 tesB (PVLB_03305) reverse \\
\hline SN285 & gatgccttgcCCACCCAGGAAGGTCTGATTC & TS2 tesB (PVLB_03305) forward \\
\hline SN286 & atccccgggtaccgagctcgTGGCGGCGGTGTCTGTAATG & TS2 tesB (PVLB_03305) reverse \\
\hline SN287 & TCGACTGCATGCGTCACTCC & $\begin{array}{l}\text { Verification cointegration tes } B \\
\text { forward }\end{array}$ \\
\hline SN288 & GATGGCCTTGCTGCTCATGG & $\begin{array}{l}\text { Verification cointegration tes } B \\
\text { reverse }\end{array}$ \\
\hline SN301 & agggataacagggtaatctgCACGAATCGAACGGGCAAC & TS1 fadA2 (PVLB_12575) forward \\
\hline SN302 & ccatgcgcttAACGGCTTGTTACCCAGG & TS1 fadA2 (PVLB_12575) reverse \\
\hline SN303 & acaagccgttAAGCGCATGGGTTCAGTCATC & TS2 fadA2 (PVLB_12575) forward \\
\hline SN304 & atccccgggtaccgagctcgTTATGGCGAAAGCCGCTATC & TS2 fadA2 (PVLB_12575) reverse \\
\hline
\end{tabular}




\begin{tabular}{|c|c|c|}
\hline SN307 & ACAGGCCGTAGCCATACGAG & $\begin{array}{l}\text { Verification cointegration fadA2 } \\
\text { forward }\end{array}$ \\
\hline SN308 & CACATCGGTGTCGACTAC & $\begin{array}{l}\text { Verification cointegration fadA2 } \\
\text { reverse }\end{array}$ \\
\hline BW_013 & TTTGCACTGCCGGTAGAAC & $\begin{array}{l}\text { corresponding forward primer for } \\
\text { verification reverse primer }\end{array}$ \\
\hline BW_014 & AATACGCAAACCGCCTCTC & $\begin{array}{l}\text { corresponding reverse primer for } \\
\text { verification forward primer }\end{array}$ \\
\hline \multicolumn{3}{|l|}{ Plasmids } \\
\hline SN033 & GTCTAGAGGAGCATGCGACG & $\begin{array}{l}\text { backbone for } \mathrm{pSN} 1 \text { without } \\
\text { nagR/p } \text { p }_{\text {nagAa }} \text { forward }\end{array}$ \\
\hline SN034 & GCGGTAATACGGTTATCCAC & 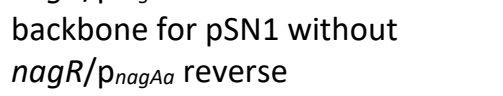 \\
\hline SN035 & gataaccgtattaccgcCCTGCGCCATCAGATCCTTG & forward pEG1675 \\
\hline SN070 & gcatgctcctctagacGAAGCCGCACGTCATCTAGC & reverse pEG1675 \\
\hline SN071 & ATTCGCCCTATAGTGAGTCG & $\begin{array}{l}\text { backbone for pSN2 without } \\
\text { nagR/p pagAa forward }\end{array}$ \\
\hline SN072 & AGCGTAATGCTCTGCCAGTG & $\begin{array}{l}\text { backbone for } \mathrm{pSN} 2 \text {, without } \\
\text { nagR/pnagAa reverse }\end{array}$ \\
\hline SN073 & ggcagagcattacgctCACGACACGCGTCATTAATC & forward pEG1675 \\
\hline SN074 & ctcactatagggcgaatCCTTTGAGTGAGCTGATACC & reverse pEG1675 \\
\hline \multicolumn{3}{|c|}{ Genome integration } \\
\hline SN250 & CTAGTCTTGGACTCCTGTTGATAGATCC & pTn backbone forward \\
\hline SN251 & $\begin{array}{l}\text { CCTGTTCGGTTCGTAAACTGTAATGCAAGTAGCGTATGCG } \\
\text { C }\end{array}$ & pTn backbone reverse \\
\hline SN252 & cattacagtttacgaaccgaacaggCCTGCGCCATCAGATCCTTG & $\begin{array}{l}\text { fadR-fadD-fadM fwd, overlaps to } \\
\text { pTn7-MK backbone }\end{array}$ \\
\hline SN253 & tctatcaacaggagtccaagactagCCTTTGAGTGAGCTGATACCG & $\begin{array}{l}\text { 'tesA-fadB-co_aco rev, overlaps to } \\
\text { pTn7-MK backbone }\end{array}$ \\
\hline SN254 & gattaatgacgcgtgtcgtgGAAGCCGCACGTCATCTAG & $\begin{array}{l}\text { fadR-co_fadD-fadM rev, overlaps } \\
\text { to 'tesA-fadB-co_aco }\end{array}$ \\
\hline SN255 & gacgtgcggcttcCACGACACGCGTCATTAATC & $\begin{array}{l}\text { 'tesA-fadB-co_aco fwd, overlaps } \\
\text { fadR-fadD-fadM }\end{array}$ \\
\hline \multicolumn{3}{|c|}{ Sequencing primers } \\
\hline SN045 & GTTCCACTGAGCGTCAGACC & sequencing pSN1 \\
\hline SN046 & TGGCAATTCCGACGTCTTAG & sequencing pSN1 \\
\hline SN047 & GTTAAACCCGCCACCAGATG & sequencing pSN1 \\
\hline SN048 & CGATCACGCCGATGCCTTTG & sequencing pSN1 \\
\hline SN049 & TGCCGGACGCGATTTCTTTC & sequencing pSN1 \\
\hline SN050 & TCCTGGTGTCTGGCTTCAAC & sequencing pSN1 \\
\hline SN051 & CAGGGTGGTGAATGTGAAAC & sequencing pSN1 \\
\hline SN052 & GTTATATCCCGCCGTCAACC & sequencing pSN1 \\
\hline SN053 & GTAGCGGATGCGCTTATTAC & sequencing pSN1 \\
\hline SN061 & ATGCTGTCCCATTGCATGAG & sequencing pSN1 \\
\hline SN062 & GGGTATCGAGGCGGATTCAC & sequencing pSN1 \\
\hline SN063 & CGCCACCTAACAATTCGTTC & sequencing pSN1 \\
\hline SN064 & GGATGGCATGACAGTAAGAG & sequencing pSN1 \\
\hline SN054 & CGACACGCGTCATTAATCAG & sequencing pSN2 \\
\hline SN055 & CCTTGACTGGCTGGAAGATG & sequencing pSN2 \\
\hline SN056 & GGCAAAGCGAAGAAACTCAC & sequencing pSN2 \\
\hline
\end{tabular}




\begin{tabular}{lll} 
SN057 & GCCGAAGAAAGAAGAAGACG & sequencing pSN2 \\
SN058 & TCCGTCGCTGCAGATTAAAG & sequencing pSN2 \\
SN059 & GATCGCCAGGAACTGGAAAC & sequencing pSN2 \\
\hline
\end{tabular}

${ }^{a}$ Underlined nucleotides refer to Gibson Assembly overlapping regions

Supplementary Table S2. Fuel properties of the produced methyl ketone (MK) mixture

\begin{tabular}{|c|c|c|c|c|c|c|c|}
\hline MK species & C11 & C11:1 & C13 & C13:1 & C15 & C15:1 & C17:1 \\
\hline $\begin{array}{l}\text { Mol fraction (VLB120 } \Delta 3 \mathrm{pPrc} \\
{[\mathrm{mol} / \mathrm{mol}]}\end{array}$ & 0.26 & ND & 0.20 & 0.31 & 0.01 & 0.19 & 0.02 \\
\hline Derived cetane number (DCN) & 51.8 & 39.7 & 62.3 & 50.1 & 76.6 & 60.4 & 72.9 \\
\hline Boiling point $\left[{ }^{\circ} \mathrm{C}\right]$ & 224.0 & 221.7 & 255.8 & 253.6 & 282.5 & 279.8 & 306.5 \\
\hline
\end{tabular}

The derived cetane number (DCN) of a methyl ketone (MK) blend was determined by assuming linear additivity (Dahmen and Marquardt, 2017; Knop et al., 2014) and using the following equation, in which $X_{i}$ and $D C N_{i}$ designate the mole fraction and the DCN of component $i$, respectively (Dahmen and Marquardt, 2017; Knop et al., 2014):

$$
\text { Derived cetane number }_{M K \text { blend }}=\sum_{i} x_{i} \cdot \text { DCN }_{i}
$$

